Jessika Reissland, Dietrich Manzey

\title{
Serial or overlapping processing in multitasking as individual preference: Effects of stimulus preview on task switching and concurrent dual-task performance
}

Journal article | Accepted manuscript (Postprint)

This version is available at https://doi.org/10.14279/depositonce-10983

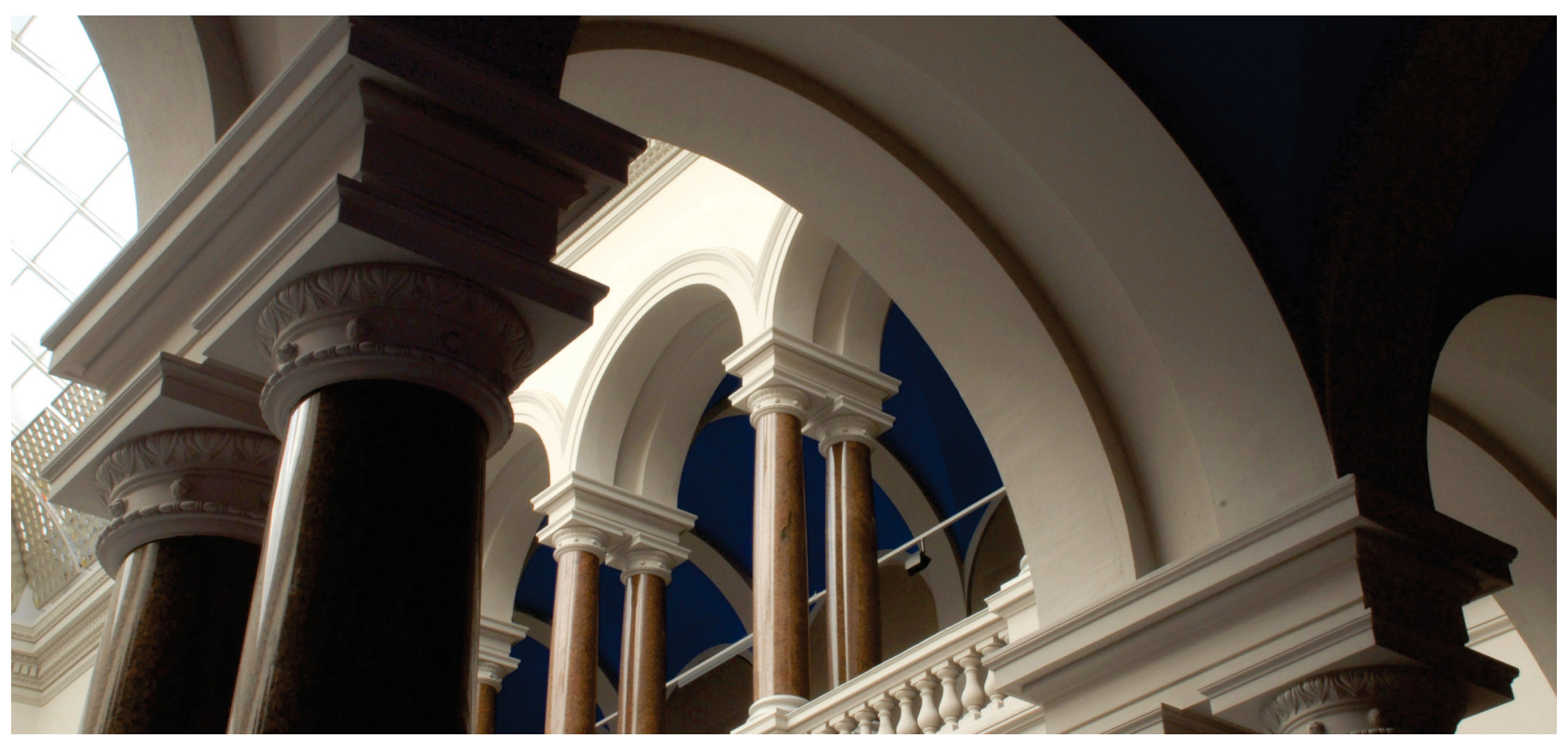

Reissland, J., \& Manzey, D. (2016). Serial or overlapping processing in multitasking as individual preference: Effects of stimulus preview on task switching and concurrent dual-task performance. Acta Psychologica, 168, 27-40. https://doi.org/10.1016/j.actpsy.2016.04.010 
Erschienen in: Acta Psychologica, 2016, Vol. 168, 27-40

Serial or overlapping processing in multitasking as individual preference: Effects of stimulus preview on task switching and concurrent dual-task performance

\section{Jessika Reissland ${ }^{\mathrm{a}} \&$ Dietrich Manzey ${ }^{\mathrm{a}, \mathrm{b}}$}

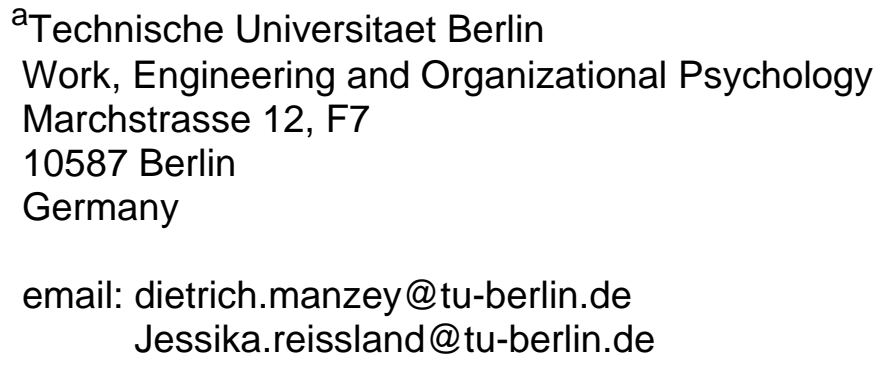

${ }^{b}$ Corresponding author. Please correspond to: dietrich.manzey@tu-berlin.de 
Abstract

Understanding the mechanisms and performance consequences of multitasking has long been in focus of scientific interest, but has been investigated by three research lines more or less isolated from each other. Studies in the fields of the psychological refractory period, task switching, and interruptions have scored with a high experimental control, but usually do not give participants many degrees of freedom to self-organize the processing of two concurrent tasks. Individual strategies as well as their impact on efficiency have mainly been neglected. Self-organized multitasking has been investigated in the field of human factors, but primarily with respect to overall performance without detailed investigation of how the tasks are processed. The current work attempts to link aspects of these research lines. All of them, explicitly or implicitly, provide hints about an individually preferred type of task organization, either more cautious trying to work strictly serially on only one task at a time or more daring with a focus on task interleaving and, if possible, also partially overlapping (parallel) processing. In two experiments we investigated different strategies of task organization and their impact on efficiency using a new measure of overall multitasking efficiency. Experiment 1 was based on a classical task switching paradigm with two classification tasks, but provided one group of participants with a stimulus preview of the task to switch to next, enabling at least partial overlapping processing. Indeed, this preview led to a reduction of switch costs and to an increase of dual-task efficiency, but only for a subgroup of participants. They obviously exploited the possibility of overlapping processing, while the others worked mainly serially. While task-sequence was externally guided in the first experiment, Experiment 2 extended the approach by giving the participants full freedom of task organization in concurrent performance of the same tasks. Fine-grained analyses of response scheduling again revealed individual differences regarding the preference for strictly serial processing vs. some sort of task interleaving and overlapping processing. However, neither group showed a striking benefit in dual-task efficiency, although the results show that the costs of multitasking can partly be compensated by overlapping processing.

Keywords: dual-task performance, task switching, task organization, preview, individual differences, switch costs

PsychINFO Classification Codes: 2300, 2323, 2340, 2346 


\section{Introduction}

In the modern work environment as well as in every-day life, we are often confronted with two or more tasks at the same time, be it programming a navigation aid while driving, reading newspaper while watching TV, or simultaneously controlling several displays in air traffic control. Such multitasking puts high demands on the human information processing system. This involves different phenomena and processes such as task switching, overlapping processing of information from different tasks, or coping with responding to two tasks in close succession or at the same time. In order to study human multitasking, several paradigms have been used, including anticipated, cued or voluntary task switching (Jersild, 1927; Kiesel, Steinhauser, Wendt, et al., 2010), the psychological-refractory-period (PRP) paradigm (Telford, 1931; Pashler, 1994a), and the concurrent dual-task paradigm (Jastrow, 1891; Navon \& Gopher, 1979). More recently, also different paradigms to study effects of enforced multitasking and task switching caused by interruptions have emerged (Trafton \& Monk, 2008).

All of these paradigms focus on somewhat different aspects of multitasking. The task switching and interruption research addresses issues of strict sequential task performance which arise when individuals have to cope with multiple task demands but cannot work on the several tasks concurrently or in parallel. This, e.g., involves situations where the tasks are presented at different locations, have the same input devices for responses, or where the human is in an overload situation. The results of this research have provided evidence for several performance costs involved in task switching mainly due to time needed for task-set reconfiguration when switching back and forth between task (Kiesel et al., 2010; Monsell, 2003) or re-activation of task-goals after unanticipated interruptions (Altman \& Trafton, 2002). Wickens, Gullwitzer \& Santamaria (2015) have provided a first model for predicting decision processes in this sort of sequential multitasking when individuals can freely decide when and for how long they perform each out of two or more tasks.

In contrast, the PRP paradigm investigates limits of overlapping processing when two discrete tasks are presented with temporal overlap. As with the task switching paradigm the main focus of this paradigm has been on detailed analyses of effects of multitasking on timing of responses when combining two relatively simple cognitive tasks. The results suggest that overlapping processing of different tasks is possible for certain stages of information processing (e.g. perceptual processes, processes of response execution) but that also a sort of central limitation exits which makes it impossible or at least difficult to perform response selection processes at the same time either due to a structural bottleneck (Pashler, 1994a) or a limited central capacity (Meyer \& Kieras, 1997; Navon \& Miller, 2002; Tombu \& Joelicur, 2003).

The concurrent dual-task paradigm has often been used in human factors research to understand how well humans can concurrently perform different tasks. In contrast to the task switching, interruptions and PRP research which focusses on basic cognitive mechanisms involved in multitasking, the paradigm has mainly been used for analyzing effects of concurrent multitasking on a more holistic performance level using relatively global performance measures of multitasking efficiency and task interference. Their results provide evidence that dual-task interference is mainly determined by the similarity of cognitive resources required by the different tasks (e.g. Navon \& Gopher, 1979; Wickens, 2002). 
The current research capitalizes on all of these different lines of research and tries to link some of the ideas and insights gained with these different paradigms. Two experimental paradigms are introduced that combine enforced task switching (experiment 1 ) and voluntary task switching (experiment 2) with a preview to the stimulus of the other task. Situations where humans have to perform several tasks concurrently with all relevant task stimuli principally available, can often be found in real-life, e.g. when answering a phone call while working on emails. Such a situations, in principle, provide the option to choose between different modes of multitasking (Wickens \& McCarley, 2008) by enabling not only to work on both tasks in a strictly serial manner but alternatively to make use of task interleaving strategies (including overlapping or parallel processing) to optimize multitasking performance. We were specifically interested in two aspects. First, to what extent humans would make use of the option of overlapping processing in such situations. Based on evidence from all lines of multitasking considered above, we expected to find individual preferences for serial vs. overlapping modes of multitasking. The second aspect relates to the impact of the use of overlapping processing with respect to the overall multitasking efficiency in this situation. We will elaborate on both aspects before turning to the actual experiments.

\subsection{Individual preferences for different modes of multitasking}

Recent research suggests that humans are in principle flexible in applying serial or overlapping strategies of task processing in multitasking situations. Most of this evidence has been accumulated from research with the PRP paradigm suggesting that performance costs arising in multitasking situation are not due to strict structural limitations but often related to strategic choices determined by different task characteristics (e.g. Janczyk, 2015; Lehle \& Huebner, 2009; Lehle, Steinhaeuser \& Huebner, 2009; Miller, Ulrich, \& Rolke, 2009; Ruiz Fernández, Leonhard, Rolke, \& Ulrich, 2011; see for a review Fischer \& Plessow, 2015). However, beyond this, there is more, albeit not very systematic evidence from all different lines of multitasking research suggesting that individuals might differ with respect to whether they "naturally" prefer a serial or an overlapping mode of multitasking.

First evidence goes back to the original work on task switching by Jersild (1927). Without having computers, the sequence of two verbal tasks was presented to the participants in form of printed lists including, intentionally or not, the option of a preview of the upcoming task stimuli. In contrast to the very robust finding of switch costs in more recent research (Monsell, 2003), Jersild (1927) pointed out a considerable variance in the data due to "... the fact that some subjects made a gain, others a loss in doing the shift test." (p. 23). Jersild (1927, p. 24) suggested that the overall gains achieved by a subgroup of participants were due to overlapping processing, i.e. a processing of the stimulus of the next task, while still executing the response to current task stimulus. This suggests that, whereas some participants have used the preview option to optimize their performance, others obviously did not use this option and worked in a strictly serial manner on the two alternating tasks. However, this anecdotal observation was not analyzed further in a systematic way. Spector \& Biedermann (1976; Exp. 1) replicated the benefits of a preview option but did not look at individual differences in using it. In addition, they treated the availability of preview just as a disturbing factor that can mask switch costs and did not provide it in their succeeding experiments. This has set the ground for the subsequent task switching research which never again has addressed the effects of preview and possible individual differences in using it. 
A second set of evidence includes observations from PRP research, particularly in studies where participants got intensive practice and/or were provided with some degrees of freedom about how and in what sequence to respond to two simple tasks presented simultaneously or in short succession. For example, Schumacher and colleagues assessed how the typically observed prolongation of the response time to the second task when presented in very short succession to the first one, would be affected by extensive task practice (Schumacher, Seymour, Glass, et al., 2001). Their results provide evidence that task practice can result in "virtually perfect time-sharing" without any response slack. Yet, this effect only emerged for a subgroup of five (out of 11) participants who were responsible for the overall statistical significance. Schumacher et al. (2001) attribute this finding to individuals differing in their "personal preference for cautious or daring task scheduling" (p. 105), with the former strategy characterized by "minimal overlap in processing for the two tasks" and the latter one characterized by a "great deal of processing overlap" (p. 107). On a theoretical level, the idea of such differences has been incorporated in models which describe the central bottleneck as a strategic instead of a structural one (e.g. Meyer, Kieras, Lauber, et al., 1995). Related evidence from the PRP paradigm includes the common finding that often 10-30\% of participants do not work strictly serially on the two tasks but tend to group their responses (e.g. Pashler \& Johnston, 1989; Ulrich \& Miller, 2008; Schubert, 1996). Though those "response groupers" do not necessarily show indications of overlapping processing, they seem to interleave both tasks instead of processing them in strictly serial order. Remarkably, the percentage of response groupers usually increases in conditions where the presented task sequence remains ambiguous due to very short or no stimulus onset asynchronies implicating a higher degree of freedom for self-organization with respect to the response order (e.g. Pashler, 1994b; Pashler \& Johnston, 1989).

Finally, some direct evidence for individual preferences for serial vs. overlapping modes of processing in multitasking situations stems from early research of Damos and colleagues (Damos \& Wickens, 1980; Damos, Smist \& Bittner, 1983). In a classical concurrent dual-task paradigm, participants had to concurrently perform two simple discrete tasks in a completely self-organized manner, i.e. without any prescription how to organize their task processing. Analyzing the time structure of response sequences, Damos and Wickens (1980) found six out of 16 participants using a strategy of overlapping processing or task interleaving while another eight participants worked on the tasks in a strictly serial manner. Another study of Damos et al. (1983) confirmed this general finding of individual differences based on a larger number of participants.

Altogether, these observations drawn from different lines of multitasking research consistently point to the existence of two types of multitaskers: those who attempt to process multiple tasks as separated from each other as possible, and those who try to work on them in an interleaving or even overlapping manner. The fact that the supporting findings were reported from a large range of different paradigms points to the importance of such a distinction to understand human multitasking. However, the observations stemming from the task switching and PRP literature usually were considered rather as a "side product" or even as disturbing factor (e.g. response grouping, Ulrich \& Miller, 2008) and have not been further analyzed or discussed in a systematic way, thus far. The early work of Damos and colleagues has just remained a sort of pilot research and has also not been followed up in later work. As a consequence, our first research question will address to what extent options of overlapping processing provided in a task switching paradigm will be used and how individuals will differ in their preference for one or the other mode of multitasking. 


\subsection{Impact of different strategies of task processing on overall multitasking efficiency}

Our second research question addressed how the different proposed strategies of task processing would affect the overall multitasking performance in terms of multitasking efficiency. The general issue of serial versus parallel processing has always been the main driver of PRP research. Recent work, adopting the view of a strategic rather than a structural bottleneck, has specifically discussed the relative advantages of serial versus parallel processing with respect to performance costs involved in multitasking (e.g., Lehle et al., 2009; Miller et al., 2009; Ruiz Fernández et al., 2011). Based on an assessment of the total response time (TRT), i.e. the sum of the mean response times of both tasks and comparing it with the respective single-task response times, usually costs of multitasking are found which are higher for parallel then serial processing, with a reverse finding only in situations when the two tasks are presented in very short succession (Ulrich \& Miller, 2008). However, while the TRT is certainly suitable to evaluate the effects of serial versus parallel processing in the stage of response selection, it does not necessarily reflect the overall net performance effects of the holistic multitasking situation.

This can be illustrated by the following consideration that is based on a typical result from PRP research (e.g. Pashler, 1994a), but can be transferred to other multitasking paradigms as well: Consider a participant responding to two choice-reaction tasks, T1 and T2, which are presented with temporal overlap, e.g. with a stimulus onset asynchrony (SOA) of 50ms. While mean response times (RT) for each task are 500ms in a single-task condition, presenting the two tasks in close succession leads to a typical PRP effect, i.e. the time to respond to T1 remains unaffected but the response to T2 prolongs to $700 \mathrm{~ms}$. When just looking at the component tasks T1 and T2 in isolation, the time to respond to T2, and thus also the TRT, has increased by $200 \mathrm{~ms}$ (i.e., single task: RT1 + RT2 $=500 \mathrm{~ms}+500 \mathrm{~ms}=$ $1000 \mathrm{~ms}$; dual-task: RT1 $+\mathrm{RT} 2=500 \mathrm{~ms}+700 \mathrm{~ms}=1.200 \mathrm{~ms})$. This reflects the usually stated dual-task cost that can be explained by a central limitation of the response selection stage. However, a different picture emerges when the time needed for overall completion of both tasks (i.e. the time from the onset of the stimulus of T1 to the response to T2) is considered. This time amounts to only $750 \mathrm{~ms}$ (i.e. starting with the onset of T1 at Oms + SOA $+\mathrm{RT} 2=0 \mathrm{~ms}+50 \mathrm{~ms}+700 \mathrm{~ms}=750 \mathrm{~ms}$ ). Within this period of $750 \mathrm{~ms}$ both tasks have been fully processed and answered. Hence the time actually needed for processing the two tasks is at least $250 \mathrm{~ms}$ shorter than the time that would have been estimated from the single-task condition if both tasks were processed strictly sequentially (RT1 + RT2 + possible costs of switching $\geq 1000 \mathrm{~ms}$ ). Thus, the throughput of tasks, i.e. the relative number of tasks performed per given time aggregated across both tasks, would be higher than expected from the single-task performance and therefore constitutes an overall multitasking benefit.

As can be learned from this example, the conclusion whether or not dual-tasking has led to costs or benefits depends directly on how multitasking efficiency is conceptualized. Whereas the TRT measure just reflects the costs involved in multitasking related to the response selection stage (which is of main interest in PRP research), the second measure represents a net measure which charges costs of multitasking arising from a presumed central bottleneck up against benefits due to overlapping processing on other stages. Admittedly, the exact contribution of costs and benefits of multitasking is concealed in the latter measure. However, at the same time it seems to reveal a more appropriate holistic view on the overall efficiency of multitasking even in the PRP paradigm. 


\subsection{The current research}

Two experiments will be presented. The first experiment introduces the modified task switching paradigm by comparing the effects of classical serial task switching with effects of task switching modified with preview. In Experiment 2 we extend the approach by combining the preview option with a voluntary task switching paradigm. The resulting paradigm provides the opportunity to analyze performance strategies applied in completely self-organized performance of the same two tasks that were used in Experiment 1.

\section{Experiment 1}

Classical task switching paradigms enforces participants to process tasks in a strictly serial manner, although the task switches themselves might be anticipated or cued (see for different variants of the paradigm, Kiesel et al. 2010). In this case, the RT is typically slower on trials including a task switch than on trials in which the task is repeated. These switch costs seem to mainly reflect the time needed for a task-set re-configuration (Monsell, 2003). Besides that so-called mixing costs can emerge, reflected in a prolongation of task repetitions compared to the mean RT in single-task trials (Rubin \& Meiran, 2005).

Both sorts of effects obviously constitute costs of serial multitasking compared to a situation where only one task has to be performed alone. However, the situation changes if the stimuli of the alternative task are already visible before a switch (preview option). In this case, a person has the option to either shield the actually performed task in order to avoid any disturbing effects from the other, or to use this information to already start processing the next task before switching overtly to it. In the former case the person would try to ignore the information from the other task and would still work in a serial manner on both tasks. This should result in basically the same pattern of switch and mixing costs as in the classical task switching paradigm. However, in the latter case, the person might either profit from the preview by partially compensating switching costs with the time-savings achieved from overlapping processing or might adversely be affected by a confusion and/or cross-talk of the two tasks (Navon \& Miller, 1987). Anecdotal evidence from the early studies of Jersild (1927) and Spector and Biederman (1976) already suggests that preview allowing for overlapping processing was used by at least a subgroup of participants to optimize performance in a task switching setting. More direct empirical evidence that a preview can be used for overlapping processing and performance improvement comes from studies of serial reaction times and typing. For example, Pashler (1994c) had subjects carry out a self-paced serial task on letters, with and without preview. The rate of responding in the preview condition was greater than in the non-preview condition; an effect that he explained by use of overlapping processing. Similarly, preview in studies on copy-typing have shown a speed-up of typing speed which also have been taken as evidence for performance optimization through some sort of overlapping processing (Bosman, 1993; Salthouse, 1985).

The first experiment compared task switching performance with and without preview. Using two tasks with univalent stimuli (letters vs. digits) and univalent responses (left vs. right hand) issues of task ambiguity usually leading to mixing costs were minimized (Rubin \& Meiran, 2005). We expected that providing the preview-option would enable a partial or even full compensation of the switch costs by the gains achieved with overlapping processing. This should be reflected in less time losses compared to a classical (non-preview) task switching situation in which serial processing is enforced. However, this net benefit should only be 
observable in a subgroup of participants that have a preference for overlapping processing when coping with multiple task demands, i.e. can be clearly distinguished by their response behavior from participants working strictly serially on both tasks In principle, it might be possible that participants who use the preview option even yield an overall benefit of multitasking indicated by performing more tasks in the task switching condition than expected from the respective performance in the single-task condition. Such an effect would challenge or at least put into perspective the often stated disadvantages of multitasking.

\subsection{Method}

\subsubsection{Participants}

Thirty-two participants (15 female) volunteered in this study (mean age 25.3 years, $S D=4.1$; range 18-36, all right-handed). Participation was compensated with money or course credit. Additionally, every participant earned extra money for each correctly answered stimulus. Because participants worked on the different tasks for fixed time periods, this sort of compensation equally motivated speed and accuracy. Prior to the experiment, informed written consent according to the Declaration of Helsinki was obtained from each participant.

\subsubsection{Stimuli and apparatus}

We used two simple choice-reaction component tasks, a digit classification task and a letter classification task. The digit task consisted of a set of digits that should be classified according to their parity $(2,4,6$, or 8 vs. $3,5,7$, or 9$)$. The letter task comprised a set of letters that should be classified either as consonants (G, K, M, or R) or as vowels (A, E, I, or $U)$. The stimuli were displayed on an Acer LCD screen (1280 x $1024 \mathrm{Px}$, sampling with 60 $\mathrm{Hz})$ in dark blue $(R G B=245,245,245$; font size $=24 \mathrm{px})$ on light gray background $(R G B=$ $80,80,80)$. Participants responded by pressing predefined letters on a standard USB keyboard: $s=$ "even" and $a=$ "odd" with the index and middle finger of the left hand for the digit task, $k=$ "consonant" and $I=$ "vowel" with the index and middle finger of the right hand for the letter task; task-hand assignment was counterbalanced among participants. The letters were marked on the keyboard with color points for easier recognition. Task stimuli were visible until a response was recorded. Immediately after a given response, a new stimulus of the answered task was presented (response-stimulus interval $=0 \mathrm{~ms}$ ). Timing and stimulus presentation were controlled by a custom-made JAVA software running on an Intel Pentium (2.9 GHz, 8 GB RAM; Windows 7 Pro).

\subsubsection{Design}

The participants were randomly assigned to two equally sized groups. The first group performed the mixed blocks analogue to classical task switching experiments without preview. The second group was provided with a preview of the following task stimulus they had to switch to in the mixed blocks. A typical experimental run consisted of one single-task block per task, i.e. digit classification (D) and letter classification (L), and one mixed block. Task presentation in mixed blocks followed a predictable task switching sequence with three trials of each task in a row before switching to the other (i.e. ..DDDLLLDDD..). This permits to distinguish between three types of mixed-block trials: switch trials (first trial after task switch: ...DDLLLDDD..), pure repetition trials (trials preceded and followed by the same task: ..DLLLDDDL..), and pre-switch repetition trials (trials immediately preceding a task switch: ..DDDLLLD..). In addition, it makes it possible to analyze the effects of preview and possible overlapping processing in sufficient detail. 
Overall, this resulted in a 2(condition: non-preview, preview) $\times 2$ (task: letter, digit) $\times 4$ (trial type: single-task block trials, mixed block pure repetition trials, mixed block pre-switch trials, mixed block switch trials) experimental design with condition as between-subjects factor and task and trial type as within-subjects factors. However, for the statistical analyses all data were collapsed across the two highly similar tasks.

\subsubsection{Procedure}

One to three participants were tested at the same time at independent PC work stations. After a questionnaire obtaining biographical information, written instructions for the experiment were provided on the screen and could be worked through self-paced. Both tasks were first explained individually before the task switching condition was described. Each explanation included a one-minute familiarization block. Subsequently, participants received another one-minute training block on each task alone to account for initially strong practice effects in the single tasks. The actual experimental data collection followed, structured in a total of four experimental runs, each including one mixed block which lasted two minutes and two single-task blocks which lasted one minute each.
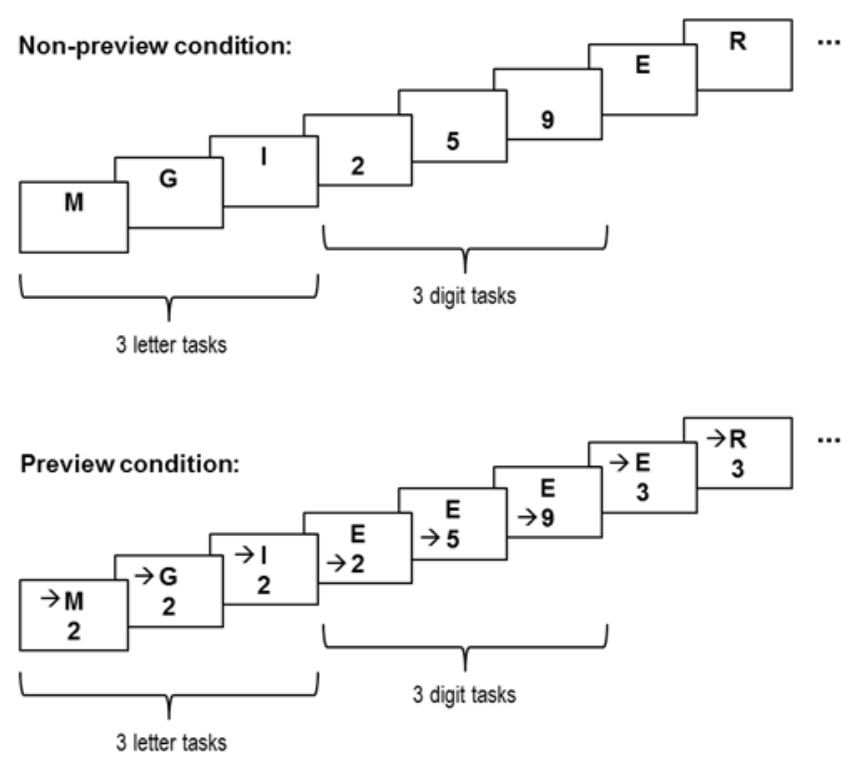

Figure 1. Examples for stimulus presentation in the non-preview and preview condition of experiment 1 . Note that in the preview-condition, the stimulus cued by the arrow always represented the stimulus of the active task to be responded to while the other stimulus provided the preview to the task to be performed after the switch.

In the single-task blocks, the participants encountered a series of stimuli, which were randomly drawn from the stimulus sets of the respective tasks, with the constraint that no stimulus would directly be repeated and that the dichotomous responses per task were equally distributed. These blocks were included in order to control for the stability of singletask performance. In the mixed blocks, the participants encountered both tasks in the predictive order as described above with the presentation of the specific task stimuli for each task following the same random schema. In the non-preview condition only the stimulus of the active task was presented (see Figure 1, upper panel). In the preview condition, each mixed block started with the presentation of stimuli of both tasks. The task stimuli were always displayed above each other in close spatial distance $\left(0.48^{\circ}\right)$ to ensure that concurrent perception was possible without eye movements. The task to be performed was prompted by a cue (arrow) in order to prevent working memory involvement of tracking the task sequence which could have confounded the comparison of conditions in a critical way. Upon 
responding, the stimulus of the active task was immediately replaced by a new stimulus, while the stimulus of the non-active task remained, enabling the participants to see the stimulus of the task which they would have to switch to when prompted. Hence, the arrow always cued the stimulus of the active task, while the stimulus without the arrow provided the preview to the other task (see Figure 1, lower panel). Participants in the preview group were able to apply different strategies of task processing. However, they did not receive any specific instruction in this respect.

Each block ended immediately if time was up. The screen turned grey and a summative performance feedback for the respective block was provided for five seconds to the participants, including the number of processed trials and the number and percentage of correct responses of both tasks. The next block could be started self-paced. Between the four experimental runs, a forced break of one minute was scheduled before the next block could be started self-paced. Overall, the experiment lasted about 45 minutes, including time for instruction and task familiarization.

\subsubsection{Data analysis}

The first of the four experimental runs was considered as training and was not analyzed. Data of the remaining runs were collapsed. This led to an average of 257 single-task trials $(\mathrm{SD}=31.94)$ and 233 dual-task block trials $(\mathrm{SD}=37.76)$ per task and participant. For each block, trials with an RT slower than three standard deviations from the participant's mean RT of this block were discarded $(2.2 \%$ of trials $(S D=0.8 \%)$ per participant on average). For the analyses of RT only correct responses were taken into account.

The data analysis was conducted in three steps: (1) a comparison of RT and error rates (ER) for the different trial types and an analysis of switch and mixing costs on a group level (nonpreview vs. preview), (2) an analysis of the individual response pattern of participants in the preview group, and (3) an examination of the impact of the preferred response pattern in the preview-group on overall multitasking performance as compared to the non-preview group.

Switch and mixing costs on group level. Switch and mixing costs in mixed blocks are usually calculated on the basis of RT and ER (see e.g. Monsell, 2003). For the non-preview condition, the RT always equaled the stimulus-response interval. However, in the preview condition the stimulus of a switch trial was always visible before actually requiring a response. Therefore we defined the RT for switch trials as the time between the cue change indicating a task switch and the respective response to the stimulus of the new task. ERs were generally determined by the rate of false responses per block. Switch costs were derived as the differences between means of RT/ER of switch trials and means of RT/ER of pure repetition trials. The latter were used as reference to prevent influences of a preceding or succeeding switch. Mixing costs were defined by the difference between means of RT/ER of pure repetition trials in mixed blocks and means of RT/ER of single-task trials.

Individual response pattern in the preview group. In order to classify participants with respect to their preferred mode of multitasking (serial versus overlapping) we used a fine-grained analysis of the overtly observable response patterns based on the following consideration: While single-task trial RTs reflect the processing time needed if only one task-set has to be held active, the RT of a switch trial includes this processing time plus at least some amount of time needed for task-set reconfiguration (Monsell, 2003). Hence, if the RT in a switch trial actually is faster than the typical RT in single-task trials, at least some processing must have taken place before the switch, i.e. in parallel to the processing of the preceding trials of the 
other task. To determine the single-task RT against which the switch trial RT would be compared, we used the range of the first quartile (quickest $25 \%$ of RTs) in the respective single-task block. On a single-trial basis, each switch trial was compared to this range and rated as fast switch if the RT was within this range or shorter. Only correct responses were included in this analysis. Note that this approach provided a relatively conservative estimation of instances of overlapping processing for each individual participant.

In order to further control for random effects we also assessed the rate of fast switches according to the criterion described above in the non-preview group. Because in this group the occurrence of a fast switch cannot result from overlapping processing, they must have occurred randomly. Only those participants in the preview group, who showed a rate of fast switches that was clearly higher than random (i.e. larger than the maximum rate observed for the participants in the non-preview group), were eventually classified as overlapping processors. All other participants in the preview group were classified as serial processors.

Overall multitasking performance. The general logic of an overall measure of multitasking efficiency has been described in 1.1. The specific measure used in our research was based on simple throughput measures, i.e. a count of number of tasks our participants could correctly perform in the given time. This has the advantage to be sensitive to variations in both speed and accuracy, and to provide a performance measure that is positively correlated with performance (the higher the better). Moreover it makes it possible to describe the overall net efficiency achieved in performing two discrete tasks concurrently in a straightforward way and has been used particularly in dual-task paradigms which provide options for selforganized task processing (e.g. Wickens, Mountford \& Schreiner, 1981) .

To better understand this measure, consider the following Gedankenexperiment: In our experiment we have worked with single-task blocks of one minute each and mixed-blocks of two minutes. Assume a participant from the "preview group" can correctly solve 42 trials of the letter task and 38 trials of the digit task in the single-task conditions. If this participant now has to work on both tasks for two minutes in mixed blocks and would be able to perform the tasks with the same throughput as in the single-task blocks (considering neither any mixing or switch costs, nor benefits from overlapping processing), we would expect 42 correct responses (CR) to the letter task and $38 \mathrm{CR}$ to the digit task, i.e. $80 \mathrm{CR}$ in total. In this case, the overall number of $\mathrm{CR}$ achieved in the dual-task condition would directly correspond to the overall number of $\mathrm{CR}$ achieved in the two single-task blocks, given that the expended time is taken into consideration. That is, the throughput for both tasks would have remained the same in both single-task and mixed conditions. Hence, the multitasking situation - in this case the task switching setting - would have led to neither costs nor benefits.

It is now easy to see how effects of mixing and switch costs, on the one hand, and overlapping processing, on the other hand, would be reflected in this performance measure. Mixing and switching costs would reduce the throughput of both tasks that can be achieved relative to the single-task performance; overlapping processing would increase this throughput. Thus, the overall number of $\mathrm{CR}$ achieved in mixed-blocks related to the respective single-task performance directly informs about the net effects gained from costs and benefits of task switching. If our participant would work strictly serially on both tasks, thus ignoring the preview option, only cost effects due to mixing and switching costs would be expected to emerge. If our participant would make use of the preview option and practice overlapping processing, the cost effects might become fully or at least partially compensated by the gains achieved through 
the overlapping processing, thus minimizing the net cost effects or even turning the overall performance in a visible multitasking benefit.

Following this reasoning, we defined the overall dual-task performance efficiency (ODTPE) in our experiments as follows:

$$
\text { ODTPE }=\left(\text { CTP }_{\text {Task } 1}+\mathrm{CTP}_{\text {Task 2 }}\right)-100
$$

With CTP Task1 and CTP Task2 defined as the component task performance (in \%) under task switching or dual-task conditions related to the performance in pure single-task blocks:

$$
\mathrm{CTP}_{\text {Task (i) }}=\frac{\mathrm{CR}_{\text {Task (i), dual-task block }}}{\mathrm{CR}_{\text {Task (i),single-task block }}} \times 100
$$

Based on this measure, net performance benefits of multitasking as described above are reflected in values of ODTPE $>0$. In contrast, net cost effects of multitasking are reflected in values of ODTPE $<0$.

\subsection{Results}

\subsubsection{Overall group effects}

Table 1 shows the mean RT and ER for both groups (non-preview and preview) in the singletask blocks and on repetition and switch trials in mixed block trials, as well as mean switch costs.

Table 1

\begin{tabular}{|c|c|c|c|c|}
\hline \multirow[b]{2}{*}{ Trial type } & \multicolumn{2}{|c|}{ non-preview } & \multicolumn{2}{|c|}{ preview } \\
\hline & RT & ER & RT & ER \\
\hline Single-task trials & 678 & 2.84 & 636 & 2.33 \\
\hline Mixed-block repetition trials & 677 & 1.94 & 638 & 1.44 \\
\hline Mixed-block pre-switch trials & 655 & 2.16 & 625 & 2.09 \\
\hline Mixed-block switch trials & 934 & 2.99 & 772 & 1.80 \\
\hline Switch costs & 257 & .16 & 134 & -.53 \\
\hline
\end{tabular}

Performance and switch costs by condition in Exp. 1

$R T=$ reactions times in $m s, E R=$ error rates in \%

Mean RT. Mean RT was examined in a repeated measures analysis of variance (ANOVA) with the between-subjects factor condition (non-preview, preview) and the within-subjects factor trial type (single-task block, mixed-block pure repetition, mixed-block pre-switch, mixed-block task switch). Reported $p$-values are Greenhouse-Geisser corrected if indicated. The ANOVA revealed a significant main effect of trial type, $F(1.4,90)=52.80, p<.001, \eta_{p}=$ .64), as well as a significant condition by trial type interaction, $F(1.4,90)=5.07, p=.020, \eta_{p}=$ .14. The main effect of condition just failed to reach the conventional level of significance, $F(1,30)=3.65, p=.066, \eta_{p}=.11$. The interaction effect is illustrated in Figure 2. While only 
small and almost constant mean differences emerged between the non-preview and the preview condition for mean RTs in single-tasks (-42ms), pure repetitions (-41ms) and preswitch trials (-30ms ), this difference was about four times higher for switch trials $(-160 \mathrm{~ms})$, indicating that the preview option specifically led to advantages when participants switched from one task to the other. This was also reflected in the significant reduction of mean switch costs in the preview condition compared to the non-preview condition (257 vs. 134ms), $F(1,30)=6.04, p=.020, \eta_{p}=.17$.

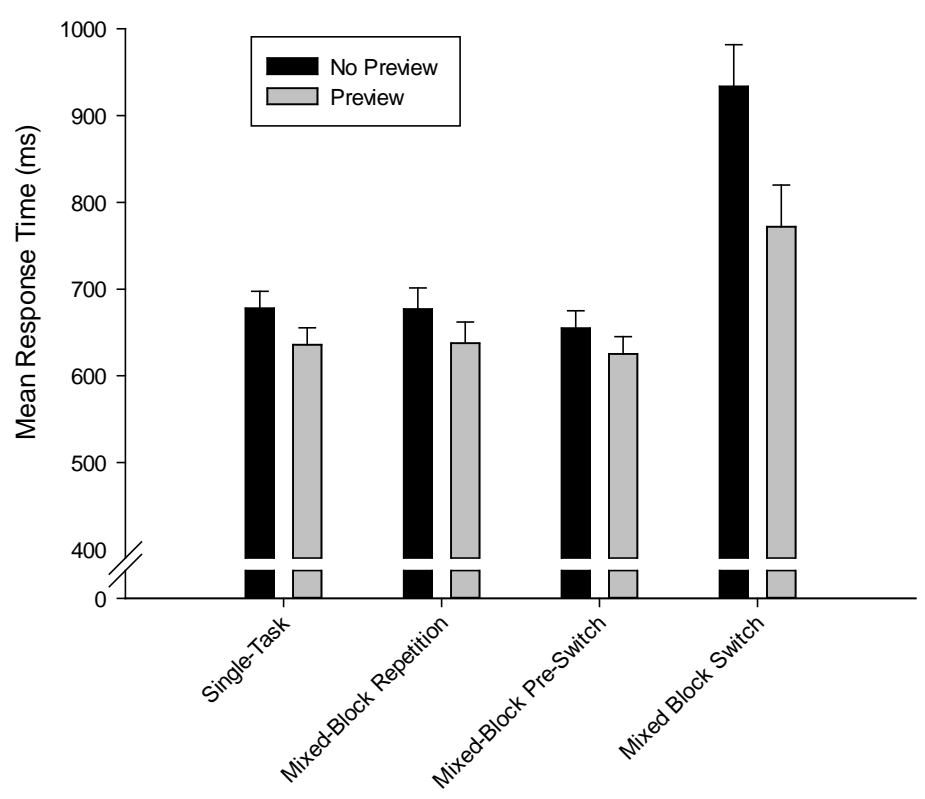

Figure 2. Mean and standard errors of response times for different types of trials (single-task, mixed-block repetition, mixed-block pre switch, and mixed-block switch trials) in the no preview and preview condition of experiment 1.

Mean error rates. The mean ER were examined in a repeated measures ANOVA analogue to the RT. Neither the main effect of condition, $F(1,30)=.60, p=.45, \eta_{p}=.02$, the main effect of trial type, $F(1.4,90)=2.09, p=.150, \eta_{p}=.07$, nor the interaction, $F(1.4,90)=.75, p=.432$, $\eta_{p}=.02$, were significant. As a consequence, also no between-group differences for switch costs emerged in the ER data, $F(1,30)=.053, p=.473, \eta_{p}=.02$.

\subsubsection{Individual response pattern}

The preferred response pattern of each participant of the preview group was determined by the fine-grained analysis of switch times described above in Section 2.1.5. In the nonpreview group an average percentage of $2.35 \%(S D=3.17 \%)$ would have been marked as fast switches ranging from $0.00 \%$ to $8.45 \%$. Therefore a rate of fast switches higher than $8.45 \%$ was considered as non-random, and only those participants of the preview group were considered as overlapping processors who showed a higher rate of fast switches than this reference. This resulted in two subgroups: $n=9$ participants with evidence for overlapping processing that is obviously higher than chance, and $n=7$ participants classified as serial processors. The mean rates of fast switches for these two subgroups were $27.62 \%$ $(S D=17.58 \%)$ and $5.55 \%(S D=3.15 \%)$, respectively. The classification of the two 
subgroups is supported by a clearly visible discontinuation in the distribution of the rate of fast switches across all 16 participants of the preview group (see Figure 3).

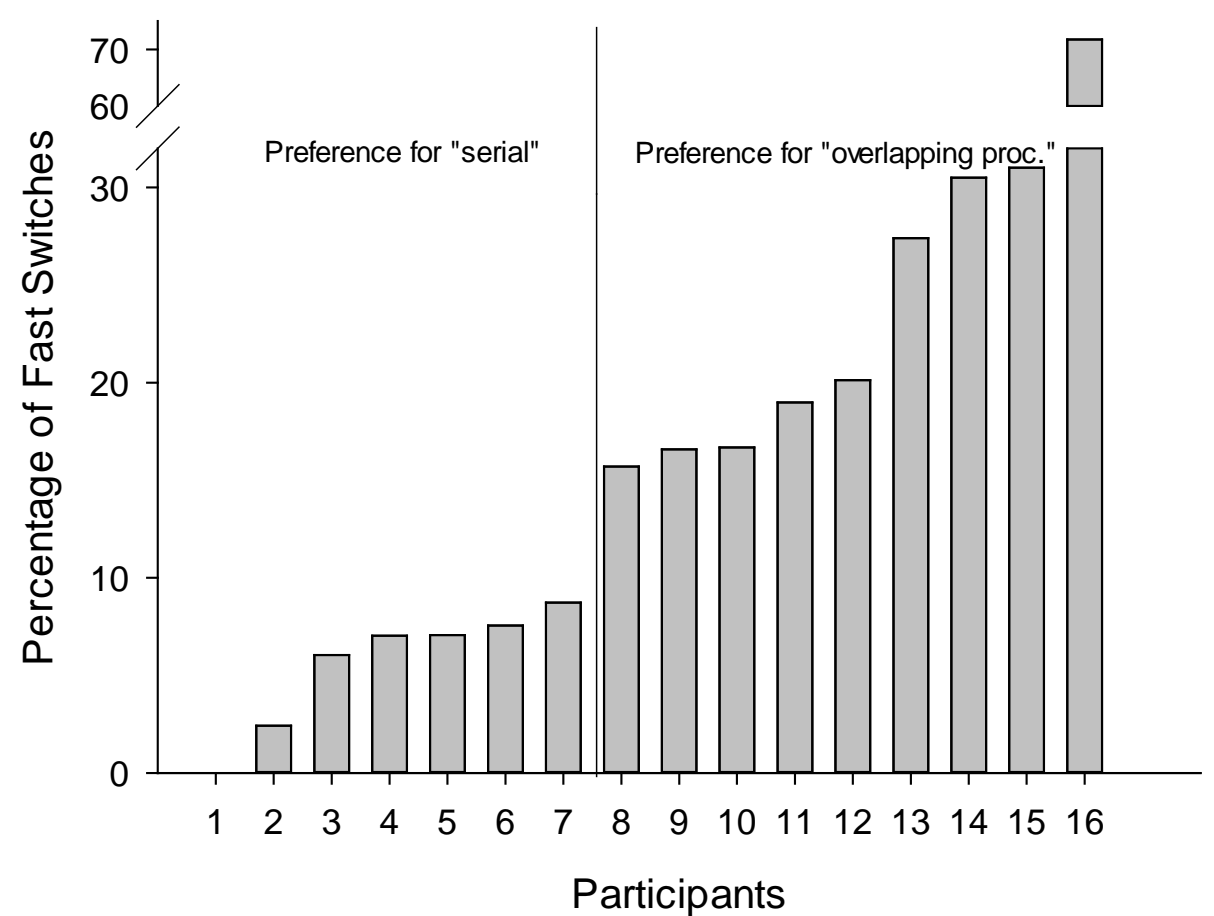

Figure 3: Distribution of percentage of fast switches across the 16 members of the preview-group.

The resulting switch costs for the non-preview group and the two subgroups of the preview condition are shown in Figure 4. Mean switch costs were almost the same for the nonpreview and the preview-serial group, but were considerably reduced to only 50ms in the preview-overlapping group. A one-way ANOVA confirmed the differences in switch costs between the three groups, $F(2,29)=8.51, p=.001, \eta_{p}=.37$. Planned non-orthogonal Sidak contrasts confirmed that the switch costs for the preview-overlapping group differed significantly from the preview-serial group $(p=.015)$ as well as from the non-preview group $(p<.001)$, whereas no significant differences emerged between the non-preview and the preview-serial group $(p=.992)$. Switch costs reflected in ER were low for all groups $(<1 \%)$ and did not differ statistically between the groups, $F(2,29)=0.71, p=.498, \eta_{p}=.05$ ).

In order to investigate whether the reduction in switch costs in the preview-overlapping group was traded-off against a prolongation of processing time in the trials preceding a task switch, we further compared the mean mixing costs reflected in RT for the two trials before the switch (i.e., mixing costs averaged across pure repetition and pre-switch trials) for the three groups. These costs were low in all groups and did not differ significantly from zero, i.e. nonpreview: $-12 \mathrm{~ms}, t(15)=-.62, p=.542$, preview-serial: $-17 \mathrm{~ms}, t(6)=-.85, p=.429$, and preview-overlapping: $6 \mathrm{~ms}, t(8)=.42, p=.688$. The analogue ER mixing costs were also low and did not differ from zero for the preview-serial group $(.62 \%, \mathrm{t}(6)=-.68, \mathrm{p}=.521)$ and the non-preview group $(-.79 \%, \mathrm{t}(15)=-2.87, \mathrm{p}=.012)$. The preview-overlapping group $(-.88 \%$, $\mathrm{t}(8)=-2.54, \mathrm{p}=.035)$ actually showed negative mixing costs in $\mathrm{ER}$, hence mixing benefits. However, these differences did not become significant when compared across groups, $\left.F(2,29)=1.22, p=.31, \eta_{p}=.08\right)$. 


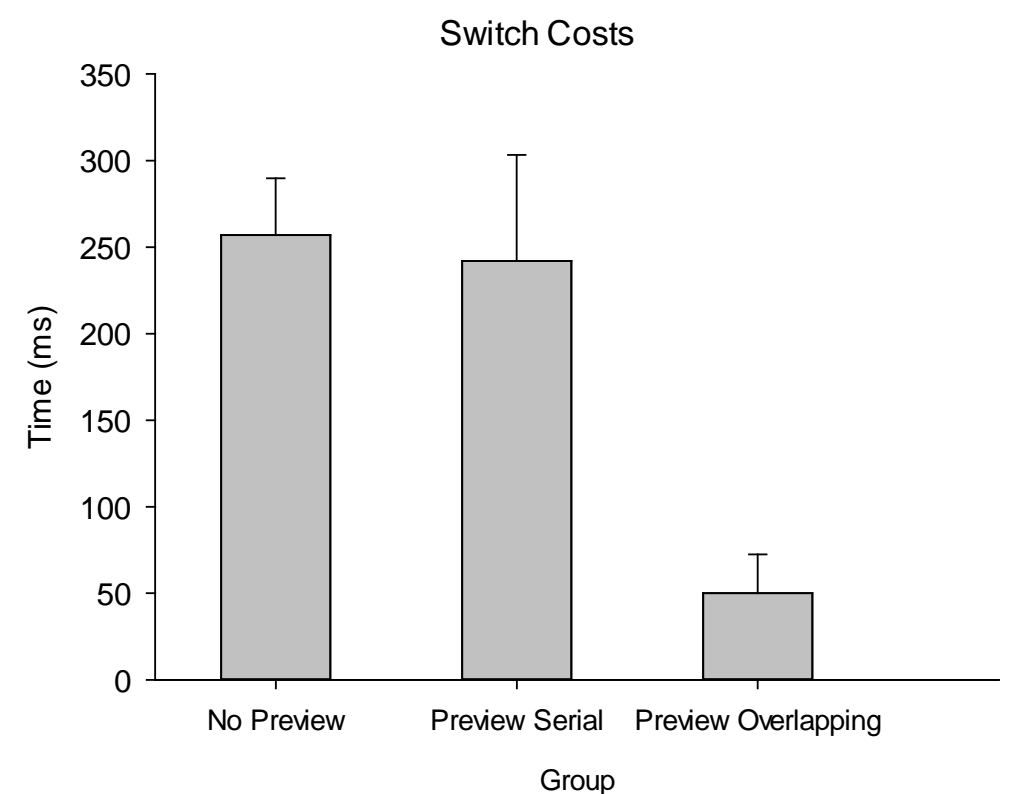

Figure 4. Mean and standard errors of switch costs averaged across the letter and digit task separately shown for participants in the non-preview condition and the two subgroups of the preview condition (serial and overlapping processors) of Experiment 1.

Being even more granular on the preview-overlapping subgroup, the mean RT of the $27.6 \%$ fast switches in mixed blocks were considerably lower than the mean RT for repetition trials (473ms vs. $620 \mathrm{~ms}, \mathrm{t}(8)=6.50, \mathrm{p}<.001$ ), resulting in a mean switch benefits of $147 \mathrm{~ms}$ associated with each fast switch. This benefit obviously was gained by some sort of overlapping processing. This becomes evident from the RT to trials of the respective other task immediately preceding a fast switch which was not compensatory prolonged but even slightly shorter compared to the mean RT for pure repetitions (604 vs. $620 \mathrm{~ms}, \mathrm{t}(8)=-1.51, \mathrm{p}$ $=.171)$. A respective analysis of the ER did not indicate any speed-accuracy trade-offs.

\subsubsection{Impact of response pattern on overall performance}

The mean ODTPE scores for the non-preview group and the two subgroups of the preview condition are shown in Figure 5. As expected the ODTPE of the serial processors $(-21.89 \%$, $S E=3.56 \%)$ was comparable to the ODTPE of the non-preview group $(-20.60 \%, S E=$ $5.67 \%)$. In contrast, the ODTPE of those participants of the preview condition who showed a response pattern indicating overlapping processing, was much better $(-9.7 \%$, SE $=3.12 \%)$, pointing to considerably reduced performance costs and, thus a better dual-task efficiency. Contrasting the performance of the two subgroups of the preview condition by a MannWhitney U-Test (because of unknown distribution characteristics) revealed a significant difference, $U=53, p<.023$. 
Overall Performance Efficiency

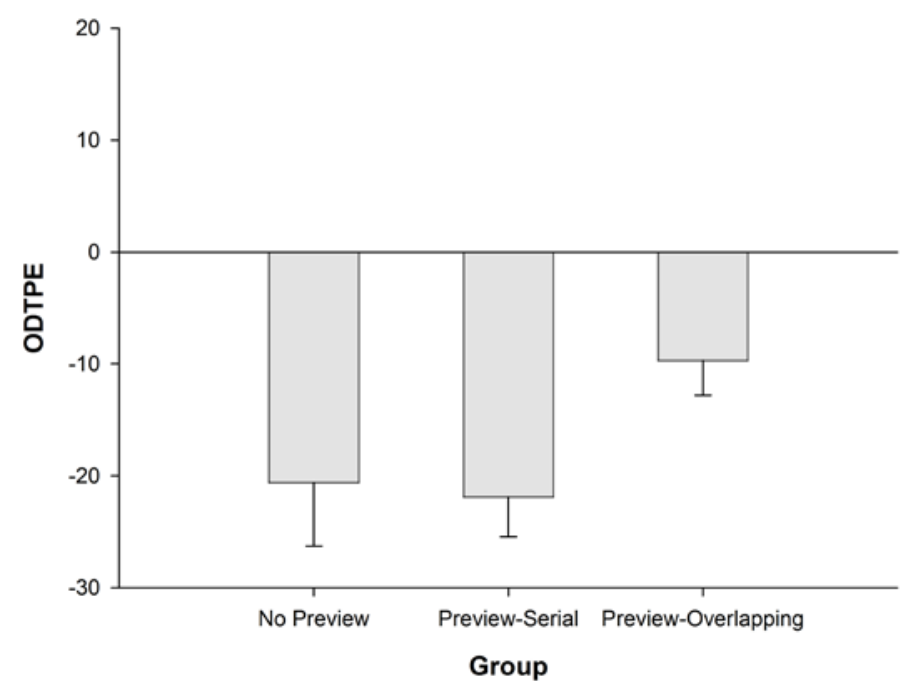

Figure 5. Mean and standard errors of overall performance efficiency (ODTPE) in mixed trials of Experiment 1, separately shown for participants in the non-preview condition and the two subgroups (serial and overlapping processors) in Experiment 1.

\subsection{Discussion}

The aim of the first experiment was to investigate whether a complete stimulus preview of the task one has to switch to would be used in a task switching paradigm to optimize performance. Because such a preview offers options for overlapping processing of the two tasks, we hypothesized that using this option would at least partly compensate the overall switch costs usually observed in task switching studies. Moreover, based on early observations reported by Jersild (1927) and other evidence suggesting that humans differ in their preference for serial vs. overlapping processing in multitasking situations, we assumed to find a similar difference. The results of the first experiment provide support for our hypotheses.

As expected, with the preview option the net switch costs could considerably be reduced, i.e. mean switch costs were only about half the switch costs of the group without preview. These gains emerged without a corresponding prolongation of mixing costs in the trials preceding a switch. This confirms a partly compensation of costs involved in task switching (e.g. due to demands of task-set re-configuration) by time-savings due to the use of overlapping processing. However, a closer inspection of the data of the preview group, revealed that this strategy of overlapping processing was applied only by a subgroup of nine participants who showed indications of overlapping processing in at least a considerable number of all switch trials (in about a third on average). The other seven participants did not show evidence for overlapping processing and, thus, were classified as serial processors. This classification was supported by the distribution of the rate of fast switches across participants that show a relatively clear cut into the two groups. It is further in line with the non-preview and previewserial group showing essentially same patterns of switch costs and overall net costs as reflected in the ODTPE (about 20\% performance decrement in mixed blocks compared to single-task blocks). In comparison, the overlapping processors were able to cut in half the costs of multitasking (ODTPE $=9.7 \%$ ). 
However, the specific and exact nature of the overlapping processing that has led to this advantage compared to strictly serial processing cannot be determined conclusively from the current data. Yet, given that the preview reduced the cost effects of task switching in the group of overlapping processors by about $200 \mathrm{~ms}$, it seems likely that at least two sorts of overlapping processing must have contributed to this overall effect. First, the full preview of the stimulus of the task to switch to next conceivably enabled participants to already encode this stimulus while still working on the previous trial. This would mean that the stimulus of an upcoming task might be processed while the other task-set is still active. Second, processes of task-set reconfiguration might already be initiated while still executing the response of the preceding trial. Both of these effects would fit to the general assumption of central bottleneck models that processes preceding and following the response selection stage can run in parallel with other processes (Pashler, 1994a, 2000). The latter effect would also directly correspond to Jersild (1927) who suggested exactly such an overlap as partial explanation for the task switching gains observed in some of his experiments.

Taken together, the results of the first experiment provide evidence that the availability of a preview option in a task switching setting, can improve the multitasking efficiency considerably if used. However, even the participants classified as overlapping processors were not able to fully reduce the performance costs associated with this sort of multitasking or even to turn overall task switching costs into net benefits. An explanation for this becomes evident from considering the rates of fast switches in this group. Obviously also these participants did not apply overlapping processing in every switch trial but only in a certain percentage of trials $(15.70 \%-71.57 \%)$. Even though providing the preview to the other task offered some degrees of freedom for task processing, it was still required to work on the two tasks according to a prescribed switch sequence. This might have hindered to apply overlapping processing more consequently. Higher rates of overlapping processing and, thus, even larger effects on multitasking efficiency, might be achieved by releasing the experimental restrictions even more and giving the participants the possibility of selfcontrolled task choice and switching. This was subject of the second experiment.

\section{Experiment 2}

In this experiment we investigated to what extent individuals would use the preview of the other task when having full voluntary control about task choice. This actually transfers the task switching paradigm into a concurrent dual-task paradigm that has been used to investigate effects of task characteristics on multitasking performance in human factors related research (e.g. Wickens et al., 1981; Manzey, 1993). In contrast to the usual procedure in the voluntary task switching paradigm (e.g. Arrington \& Logan, 2004, 2005), participants are not instructed to produce a random sequence of task-switches, but to concurrently perform the two tasks as best as they can without specific advice about how to achieve this. In addition, they always have a preview of the stimulus of the other task they might switch to available (analogue to Experiment 1). Such an approach has two important characteristics making it attractive in the context of the current research: First, participants are continuously challenged to organize their responses to the two concurrent tasks over time and must develop a strategy how to do that to achieve maximum performance. Second, the paradigm provides the participants always with complete access to information of both tasks and therefore, in principal, enables a task organization involving not only overlapping processing like in the first experiment, but also other forms of task interleaving (e.g. response grouping). Both of these characteristics make the demands of this paradigm more complex 
as in the first experiment, resembling complex multitasking challenges as can basically be found also outside the laboratory. However, at the same time the paradigm allows for a detailed analysis of response pattern and thus the identification of specific task organization strategies.

As has been described in 1.1, using such a concurrent dual-task paradigm has already provided evidence that humans differ in how they schedule and organize responses when coping with multitasking demands in a self-organized way but were not followed up since then (Damos \& Wickens, 1980; Damos et al., 1983). Based on these results and our own findings in experiment 1 we expected again to find at least two subgroups of participants: (1) one group preferring strictly serial processing of the tasks reflected in a sort of massed scheduling of responses to each task and minimizing the number of switches between tasks, and (2) another group making considerable use of task switches and overlapping processing around switches in order to optimize performance. However, providing complete freedom of task processing and response scheduling also other strategies involving some sort of interleaving the processing of the two tasks might emerge.

As in the first experiment, a second objective of this experiment was to investigate how serial versus overlapping processing and/or some sort of task interleaving would impact the overall multitasking efficiency as reflected in OTDPE measure. The results of previous findings are not clear in this respect. Whereas the results of Experiment 1 suggest that using overlapping processing in task switching with preview might reduce performance costs of multitasking but does not necessarily lead to performance benefits, data of Manzey (1993) from a concurrent dual-task paradigm with complete self-organization suggest that at least (small) performance benefits might be achieved by a task interleaving strategy based on response groupings combined with overlapping processing. Damos und Wickens (1980) reported a similar beneficial effect of response grouping, however, this was not confirmed by Damos et al. (1983) who did not find any performance differences between different strategies of task processing. In this experiment, only participants combining a high number of task switches with overlapping processing were theoretically expected to be able to even achieve a performance benefit of concurrent task performance, shown not only in a reduction of cost effects compared to single-task performance but in a real gain of performance efficiency as indicated by an ODTPE score above zero.

\subsection{Method}

\subsubsection{Participants}

Eighteen participants volunteered in this study ( 6 female). On average they were 25.3 years old $(S D=2.7$; range $=20-28)$. As in Experiment 1 , participation was compensated with money or course credit with an additional extra monetary incentive for each correctly answered stimulus for motivation. Prior to the experiment, informed written consent was obtained from each participant according to the Declaration of Helsinki.

\subsubsection{Apparatus and stimuli}

The same apparatus and stimulus material were used as in Experiment 1.

\subsubsection{Design}

All participants were provided with the preview of the other task and were free to decide when to work on which task in the dual-task blocks. Single-task blocks were included for comparison. Each experimental run consisted of two dual-task blocks, and two single-task 
blocks (one for each task, respectively). Subgroups of participants exhibiting different types of preferred response scheduling and task processing were identified post-hoc and served then as group variables for further analyses of the impact of the different multitasking strategies on overall dual-task efficiency.

\subsubsection{Procedure}

The procedure was very similar to that of Experiment 1: after a short questionnaire, instructions for performing the two tasks in single-task and dual-task conditions were presented in writing on screen. Each explanation included a one-minute familiarization block. With respect to the dual-task condition, participants were instructed to maximize overall performance while working on both tasks with equal priority. No instructions concerning possible approaches of task organization were given. After this, participants received another one-minute training block on each task before the actual data collection was started, structured in four experimental runs. Each run included two 2-minute dual-task blocks followed by two 1-minute single-task blocks, one for each task. The dual-task blocks resembled those of the preview group in Experiment 1 in how the stimuli were presented, but did not include a cue indicating which task should be answered. A break of at least one minute was scheduled between the training and the start of the experimental runs, and also between the four experimental runs. Continuing with the next run and starting each test block was always self-paced. All other procedural details (e.g. provision of feedback and payment) directly corresponded to the procedure in the first experiment. Altogether the experiment lasted about one hour per participant (including times for welcome, instruction and discharge).

\subsubsection{Data analysis}

The first of the four test blocks was considered as practice and was not analyzed. Data of the remaining test sessions were collapsed. This led to an average of 280 single-task trials (SD = 36.9) and 264 dual-task block trials $(S D=59.8)$ for each task and each participant. For each block, trials with a response time slower than three standard deviations from the participant's mean response time of this block were discarded (1.8\% of trials, $S D=0.6 \%$, per participant on average). Response times were assessed as inter-response intervals (IRIs) rather than $\mathrm{RT}$, because in case of a switch only the time between the actual and the previous response can be measured. In case of a task repetition the IRI equals the RT due to the responsestimulus interval of $0 \mathrm{~ms}$. For the analyses of response times only correctly answered trials were taken into account.

In the dual-task condition of Experiment 2 participants had much more degrees of freedom regarding task sequencing than in Experiment 1, which made it impossible to classify trials a priori as repetition, pre-switch or switch trials. In the extreme, a participant could choose to answer only digit stimuli in the first half of a two minute dual-task block and only letter stimuli in the second half. This would lead to repetition trials only, except for one switch trial. A calculation of switch costs would not be useful in this case. Vice versa, a participant could have alternated between the tasks every other trial, producing only switch trials, but no repetition trials. Therefore an analysis of response times on an overall group level was not considered as reasonable. Instead, we first identified the preferred approach to response organization for each participant in order to identify subgroups exhibiting similar response pattern. All further analyses were then performed on subgroup level.

Individual response pattern. The identification of the preferred response pattern in dual-task trials was done post-hoc, based on the observed response sequences and assessments of 
IRI. First, the rate of task switches related to the overall number of processed trials was calculated for each participant. All participants who switched between the two tasks in less than $10 \%$ of the trials were categorized as blockers who obviously preferred to work on the two tasks in a separated manner. Second, we analyzed to what extent those participants who preferred to switch more often than $10 \%$ would represent a homogeneous group with respect to the portion of switches. Third, we investigated to what extent participants who switched repeatedly between the two tasks would show evidence of task interleaving and overlapping processing around overtly observable switches. The latter analysis was based on a similar approach that was used in Experiment 1 with considering IRI instead of RT. That is, we first identified the rate of fast switches for each participant, defined as a switch trial IRI in the range of the $25 \%$ fastest responses in the respective single-task block for that task (see Experiment 1 for a more detailed description of a "fast switch"). If this rate was higher than the maximum rate of the non-preview group of Experiment 1, we considered this as proof that the participant has systematically applied a task interleaving strategy in a considerable number of switch trials. We then contrasted the mean IRI of these fast switches with mean IRIs for the trials immediately preceding these switches. Only if fast switches were not associated with compensatory prolonged response times for pre-switch trials we considered them indication of overlapping processing. All other cases were considered as evidence of a pure task interleaving strategy where participants internally "switched" between both tasks before eventually responding to them in close succession but without necessarily processing them in parallel.

Overall performance. The analysis of the overall performance was assessed with the ODTPE score, analogue to that described in Experiment 1 (see section 2.1.5).

\subsection{Results}

\subsubsection{Individual response pattern}

One participant had to be discarded due to very high error rates (>20\%). Based on an analysis of switch rates in the dual-task blocks three different subgroups of the remaining participants were identified with clearly different switch pattern reflecting different strategies of task organization: blockers, switchers, and alternaters. The distribution of percentages of switches across the 17 participants and prototypical examples of the different switch pattern of the three subgroups are shown in Figure 6 . Their basic characteristics in terms of switch rates and mean sequence lengths for each task are shown in Table 2. 

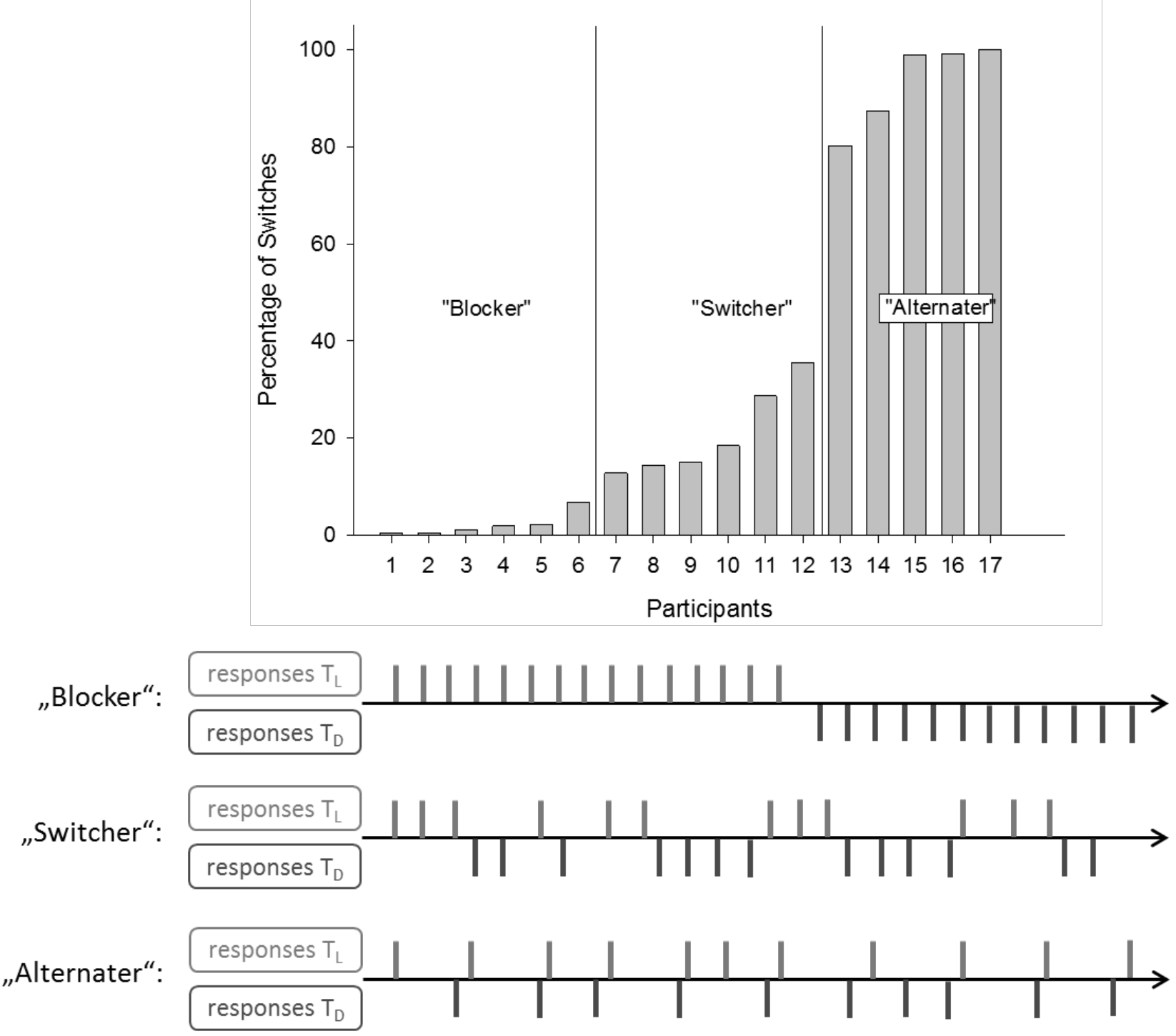

Figure 6. Distribution of percentages of switches across the 17 participants and prototypical response sequences of the three different types of task organization observed in Experiment 2.

The first subgroup included $n=6$ blockers who preferred to work on long sequences of trials of one task in a row $(>60)$ before switching to the other task, thereby minimizing the rate of switches to a mean of about $2 \%$. In contrast, participants of the second and third subgroup preferred a strategy of task organization with repeated switches between the two tasks, but differed with respect to how often and regularly task switches were made. The second subgroup (switchers) included $\mathrm{n}=6$ participants who switched repeatedly between the two tasks with a mean switch rate of about $21 \%$. The third subgroup (alternaters) included $\mathrm{n}=5$ participants who switched between the tasks mainly alternating every other trial resulting in a mean switch rate of about $93 \%$. All participants could be clearly assigned to one of the three groups. 
Table 2

Classification of task organization strategies in Experiment 2. Shown are means and standard errors (in brackets) of switch rates and sequence lengths (for digit and letter task) of the three subgroups

\begin{tabular}{lcccc}
\hline Type of Task Organization & $\mathrm{N}$ & Switch Rate & $\begin{array}{c}\text { Sequence Length } \\
\text { (Letter) }\end{array}$ & $\begin{array}{c}\text { Sequence Length } \\
\text { (Digit) }\end{array}$ \\
\hline Blocker & 6 & $2.14(.96)$ & $69.88(17.33)$ & $65.14(16.12)$ \\
Switchers & 6 & $20.84(3.75)$ & $5.60(0.85)$ & $5.38(0.74)$ \\
Alternaters & 5 & $93.23(4.03)$ & $1.09(0.05)$ & $1.09(0.05)$ \\
\hline
\end{tabular}

We assume that the subgroup of blockers preferred a serial processing strategy to cope with the multitasking demand. In order to further analyze to what extent the other two subgroups who switched repeatedly between both tasks made use of overlapping processing, we examined the number of fast switches, the mean time needed to respond to task repetitions (only possible for switchers who showed a reasonable amount of repetition trials), and the mean time needed to respond to the task immediately preceding a fast switch. Results are shown in Table 3. For comparison also mean RTs of single-task trials are shown.

Table 3

Means of inter-response intervals (IRI) and error rates (ER) of different trial types in single-task and dual-task blocks (pure repetition, pre-switch, and switch) of the three subgroups in Experiment 2 are shown. Furthermore, rate of fast switches and the respective IRI of the fast switch and the trial preceding the fast switch are shown.

\begin{tabular}{|c|c|c|c|c|c|c|}
\hline \multirow[b]{2}{*}{ Trial type } & \multicolumn{2}{|c|}{ Blocker } & \multicolumn{2}{|c|}{ Switcher } & \multicolumn{2}{|c|}{ Alternater } \\
\hline & IRI & ER & IRI & ER & IRI & ER \\
\hline Single-task trials & 589 & 4.18 & 666 & 3.58 & 583 & 2.93 \\
\hline Mixed-block repetition trials & 600 & 4.31 & 697 & 4.56 & --- & --- \\
\hline Mixed-block pre-switch trials & --- & --- & 824 & 2.20 & \multirow[b]{2}{*}{634} & \multirow[b]{2}{*}{1.35} \\
\hline Mixed-block switch trials & --- & --- & 789 & 4.82 & & \\
\hline \% Fast Switches & \multicolumn{2}{|c|}{--- } & \multicolumn{2}{|c|}{$33.4 \%$} & \multicolumn{2}{|c|}{$50.1 \%$} \\
\hline Fast switch trials & --- & --- & 274 & 4.95 & 151 & .90 \\
\hline Pre-fast switch trials & --- & --- & 1034 & 0.56 & 1192 & 1.45 \\
\hline
\end{tabular}

Because switchers provided enough events of all trial types (pure repetitions, pre-switch trials, and switch trials), we were able to calculate mixing and switch costs corresponding to those of Experiment 1 . The mixing costs for this group again were very small $(31 \mathrm{~ms})$ and did 
not significantly differ from zero, $t(5)=2.01 ; p=.100$. The same held true for the switch costs. On average they only amounted to $92 \mathrm{~ms}$ and were also not significant, $t(5)=1.83, p=$ .127. One reason for the comparatively low overall switch costs is the fact that $33.4 \%$ of all switch trials in this group were identified as fast switches (see Table 3). The mean IRIs of these switches were less than half as long as the IRIs of repetition trials, resulting in mean switch benefits of $423 \mathrm{~ms}, t(5)=-5.52, p=.003$. This benefit in the time domain did not impact the accuracy of the switchers in terms of an increase in ER $(4.95 \%$ vs. $4.56 \%, t(5)=$ $.28, p=.791)$. However, as becomes evident from a consideration of the mean IRI for preswitch trials, the putative performance benefits gained with each fast task switch were partly devoured by prolonged responses times to the tasks immediately preceding such a switch. On average these response times were prolonged by 338ms compared to a pure repetition trial. This suggests that some of the time savings reflected in fast switches were not real gains, but just related to a shift of processing time. That is, some processing of the switch trial must have taken place in a serial manner already before the response to the pre-switch trial was emitted. However, such an effect would not explain the full gain related to fast switches. The mean net benefit of $85 \mathrm{~ms}$ yielded with each fast switch $(423 \mathrm{~ms}-338 \mathrm{~ms})$ suggests that also some overlapping processing of the two tasks must have taken place during these trials.

A different picture emerged for the alternaters. In their dual-task blocks no or only very few task repetitions exist, because in an alternating sequence of task processing almost every trial is a switch trial and a pre-switch trial at the same time. As a consequence, a separate estimation of mixing and switch costs was not possible for this group. In order to better understand how the participants have processed the two tasks we again identified the fast switches which made up an amount of half of the switches (50.1\%). The mean IRIs of these fast switches amounted to only $151 \mathrm{~ms}$ on average. Thus, they were $432 \mathrm{~ms}$ shorter than the mean RTs in single-task trials, $t(4)=-5.24, p=.006$. These very fast switches again were not at the expense of accuracy, accuracy even was somewhat better than in the single-task condition (.90\% vs. $2.93 \%, t(4)=-5.99, p=.004)$. However, the mean IRIs of trials immediately preceding such a fast switch were $609 \mathrm{~ms}$ longer than the mean response times of the single-task trials. With that they were even more prolonged than in the subgroup of switchers. This suggests that alternaters did not exploit the possibility to partially overlap the processing of both tasks, but rather applied a sort of task interleaving strategy where they internally switched between the two tasks before they responded to them in close succession but without indications of parallel processing. Applying such an alternating strategy in our experiment was linked to considerable time costs which amounted to mean net costs of $177 \mathrm{~ms}$ per fast switch.

Overall performance. Figure 7 shows the resulting overall dual-task performance efficiency (ODTPE) achieved by the three subgroups. As becomes evident, all three subgroups yielded a negative ODTPE. Least dual-task costs could be observed for participants working in blocks $(-4.92 \%, S E=1.19 \%)$. The dual-task costs of switchers and alternaters were considerably higher (switchers: $-16.38 \%, S E=7.24 \%$; alternaters: $-28.12 \%, S E=14.18 \%$ ). In order to take the unknown distribution characteristic and obvious violations of variance homogeneity into account, Mood's Median Test was used to analyze the differences between the three groups statistically. This test just failed to reach the conventional level of significance $\left(c h i^{2}(2)=5.09 ; p=.078\right)$. 


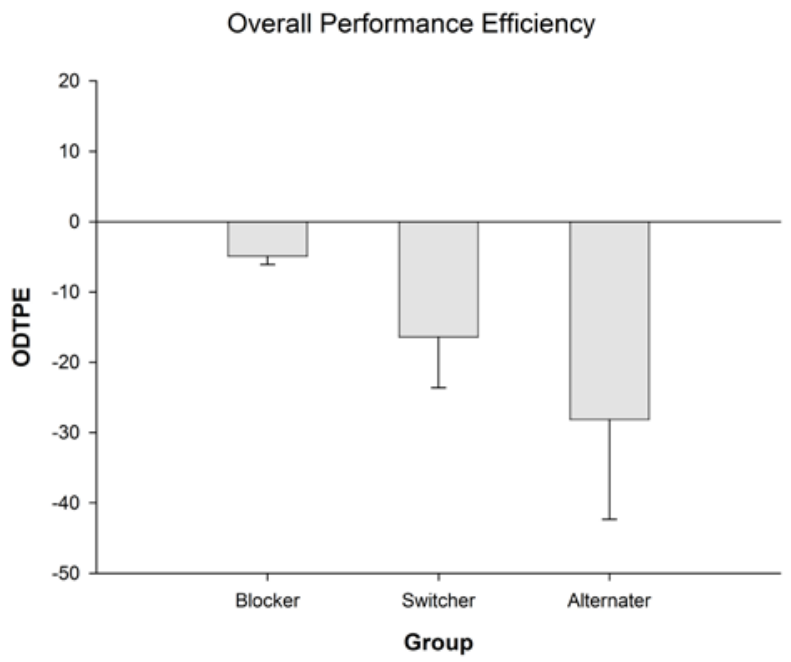

Figure 7. Mean and standard errors of overall performance efficiency in dual-task blocks of experiment 2, seperately shown for participants in the three subgroups (blockers, switchers, and alternaters).

\subsection{Discussion}

Performing two discrete cognitive tasks concurrently in a self-organized manner puts high demands on executive control and can involve all sorts of mechanisms that have been investigated separately in task switching and PRP research such as inhibition and reconfiguration of task sets and organizing response selection processes. The second experiment was conducted to analyze how individuals would deal with these demands in dual-task situations where they have considerable degrees of freedom for task organization and how this would affect their dual-task performance. As expected based on the findings of the first experiment and results of other previous research, clear evidence for individual differences with respect to preferred modes of multitasking was found. Specifically, based on a post-hoc classification of their individual response patterns three subgroups could be distinguished which we referred to as blockers, alternaters and switchers.

The group of blockers includes six (35.3\%) participants who obviously tried to minimize task switches by performing long runs of one task before eventually switching to the other. This resembles what has been called "massed" strategy by Damos and colleagues (Damos \& Wickens, 1980; Damos et al., 1983). They found that the majority of participants showing this type of task organization consistently produced more than 10 repetitions before switching. The blockers in our study even performed more than 60 trials of one task type on average. This high number of repetitions cannot be explained by the switch avoidance tendency of about $60 \%$ that is usually found in voluntary task switching (VTS) studies when asking the participants to mimic random task choice (Arrington \& Logan, 2005; Wickens et al., 2015). Instead it represents a manifestation of a preference for serial task processing, as these blockers literally try to avoid the dual-task situation by breaking it down in long sequences of single-task runs.

An obvious advantage of this strategy is that it allows for a high degree of task-shielding and, thus, for a minimization of possible cross-talk effects between tasks. In addition, only few task-set re-configurations are necessary, and demands on executive control are minimized. One reason to prefer this strategy might be related to a general avoidance of multitasking as a sort of a cognitive miser, although task-shielding processes have been supposed to be more resource-demanding than modes of parallel processing (e.g. Lehle \& Hübner, 2009; Plessow, Schade, Kirschbaum \& Fischer, 2012). Yet, the latter findings mainly stem from 
studies with the PRP paradigm where demands on task-shielding are much higher than in our paradigm. The main disadvantage of the blocking strategy is that any possibilities of parallel processing cannot be exploited and overall multitasking efficiency remains naturally limited. It is therefore not surprising that the two tasks were processed under dual-task condition with essentially the same throughput than in the single-task conditions (ODTPE = $-4.92 \%)$.

However, the majority of participants did not choose to block task performance but preferred a strategy to optimize multitasking performance which involved a more or less high number of task switches. With respect to the regularity and the number of switches they did not represent a homogenous group. Instead, one subgroup of five participants (29.4\%) consistently alternated every other trial, while the other six participants (33.6\%) switched on average every 5 or 6 trials in a rather irregular switching scheme.

A closer inspection of the response times of the alternaters revealed that every other switch trial was responded within $200 \mathrm{~ms}$ or less. However, the mean IRI to the trial immediately preceding such a fast switch indicated that these times were more than just compensatory prolonged. That indicates a high interleaving of the two tasks, but without indications of overlapping processing. The resulting pattern resembles that of response grouping sometimes found in PRP studies (Pashler, 1994b; Ulrich \& Miller, 2008). However, this strategy does not seem to be effective in reducing the overall costs involved in multitasking. Participants from this group showed a net cost effect of on average $177 \mathrm{~ms}$ with every fast switch. That nearly reaches the time range of the mean switch costs $(220 \mathrm{~ms})$ observed in the first experiment for participants of the non-preview condition who were forced to perform task switching in a strictly serial manner. This raises the question why an alternating strategy has been chosen at all. Similar to the blocking strategy constant alternating does not require online decision-making due to the fixed task sequence which can be planned in advance and thus might have reduced the cognitive effort at least subjectively. Additionally, participants who in principle prefer serial processing of multiple tasks might have chosen alternating instead of blocking because of a subjectively better compliance with the instruction to perform both tasks concurrently with equal priority.

Turning to the subgroup of switchers, it becomes evident that they also showed evidence for task interleaving, reflected in a mean portion of 33.6\% fast switches, which on average were only slightly longer than the fast switches of the alternaters. Switchers, however, did make use of overlapping processing of the two tasks reflected in net gains of response time associated with their fast switches. In contrast to the alternaters, they applied a considerable more complex strategy of task-organization, i.e. they did not shift their task sets back and forth on every other trial but kept one task set constant across a differing number of trials (on average 5-6 trials) during which the stimulus of the concurrent task was already present. Interestingly, this kind of self-organized task scheduling resembles the task presentation structure that was used in the first experiment (with runs of three tasks) where overlapping processing could also be shown for a subgroup of participants. This suggests that the longer preview of the stimulus of the other task was a necessary prerequisite for effective use of overlapping processing.

The general finding of different strategies of a serial versus some interleaving mode of task processing in self-organized dual-task performance is in line with earlier research. Manzey (1993) investigated concurrent performance of a mental arithmetic and Sternberg memorysearch task and found 18 out of 20 participants who adopted a task interleaving strategy 
based on response groupings after intensive practice. Similarly, Damos and Wickens (1980) and Damos et al. (1983) found subgroups of participants who developed a simultaneous response strategy, with responses to both tasks emitted within $100 \mathrm{~ms}$. This strategy at least resembles the alternating or switching strategy of our study.

More surprising were the results reflected in the ODTPE measure, indicating the overall net multitasking efficiency. While it is comprehensible that the participants applying a blocking strategy and the participants combining serial processing with response grouping could not realize multitasking benefits, it was not expected that the same would held true for the switchers applying overlapping processing. On first sight, this seems to contradict our finegrained analyses that revealed these participants taking advantage of at least overlapping processing. Yet, they benefited from overlapping processing in a certain portion of the switch trials only, with considerable inter-individual differences with respect to the amount of overlapping processing (according to our conservative approach of estimation: 16.11 $46.6 \%$ of switch trials). The net time gained by parallel processing in these trials could not compensate the time loss incurring in the other switch trials. This finding again corresponds to the results of the preview-overlapping subgroup in the first experiment where benefits from overlapping processing were also found in only $15.70 \%-71.57 \%$ of the switch trials. Net switch costs of about 50ms per switch still remained in that subgroup although these had been significantly reduced compared to the preview-serial subgroup. These results rule out that the instructions and the cueing in the preview condition of the first experiment had served as a demand characteristic for serial processing of the tasks. However, the question remains why benefits from overlapping processing are not exploited in a higher number of switch trials even if complete freedom for response scheduling is provided. We will return to this question in the General Discussion.

\section{General Discussion and Conclusions}

Our experiments were driven by the idea that new insights in human multitasking might be gained by using task switching paradigms that provide more degrees of freedom for selforganized task processing. The first one involved a modified task switching paradigm with univalent stimuli and responses that allowed participants to preview the specific task stimulus of the other task. Yet, the general sequence of performing the two tasks was still externally guided. In the second experiment, any constraints of response scheduling were suspended by adding options of voluntary task switching. Both experiments confirmed our expectations that individuals obviously differ in the extent to which they prefer a serial versus some sort of interleaving and/or overlapping mode of task processing when coping with multiple task demands.

This provides converging evidence for early anecdotal reports and results from task switching and dual-task research pointing to similar differences (Damos \& Wickens, 1980; Damos et al., 1983; Jersild, 1927; Schumacher et al., 2001). Specifically, the distinction between "cautious" and "daring" strategies of multitasking proposed by Schumacher et al. (2001) fits nicely to our findings in that a cautious strategy can be characterized by a strictly serial processing of tasks, whereas a daring strategy represents a much more diverse set of strategies, involving different sorts of task interleaving and/or overlapping processing. Such a distinction has important consequences for our understanding of human multitasking. It specifically suggests that choices between serial and overlapping processing do not just reflect strategic choices dependent on specific task settings or effort considerations (Lehle \& 
Huebner, 2009; Plessow \& Fischer, 2015) but that they at least to some extent also reflect a natural preference for one or the other mode of multitasking. It directly raises another question which has not been addressed in the current research but seems to be important for future investigations, namely to what extent such an individual preference might be linked to other individual differences in cognitive functions. One obvious candidate can be assumed in working memory capacity (WMC). WMC has already been suggested to be closely linked to the effectiveness of executive attention and control (Kane, Conway, Hambrick, \& Engle, 2008). Specifically, it has been found that individuals with higher working memory capacity are more resilient towards proactive interference effects in memory and are better able to solve conflicts between competing responses (Kane \& Engle, 2000, 2003). Thus, individuals with a higher working memory capacity might also be better suited to apply task interleaving strategies and overlapping processing without committing the risk of task interference. However, the differences between alternaters and switchers in our second experiment show that just applying a daring strategy of multitasking does not mean to actually benefit from overlapping processing. While the alternaters did not manifest benefits of overlapping processing, the switchers did. This suggests that a somewhat more relaxed switching routine with more time to prepare for effective interleaving of the two tasks provides a better context for overlapping processing than a fast alternating schedule.

Our experiments also addressed the question to what extent the different approaches of task organization and response scheduling, and particularly the use of overlapping processing would affect dual-task efficiency. Although less clear-cut than expected, the results of the first experiment provide evidence for successful use of task interleaving and overlapping processing to optimize task switching in terms of reduced switch costs and higher net multitasking efficiency compared to a strictly serial processing manner. A similar effect emerged at least on a descriptive level when comparing the dual-task efficiency of switchers and alternaters in the second experiment. However, neither in the first nor the second experiment the effects of overlapping processing were so strong to actually turn dual-task cost effects in dual-task benefits as would have been reflected in positive ODTPE scores. This was probably due to the fact that in both experiments overlapping processing was only applied in a limited number of trials only and hence could not fully compensate other cost effects. This certainly has prevented a more positive effect of this strategy on overall dualtask efficiency. One reason for this could be a lack of enough practice as needed to fully establish this strategy. Another reason might be the high similarity of the two tasks with respect to the specific processing resources (Wickens, 2002). This might have made task interleaving and overlapping processing particularly difficult and prone to negative side effects. However, it is remarkable that the subgroup of switchers in the second experiment nevertheless were able to realize actual time benefits associated with task switches in up to $47 \%$ of switch trials. This shows the potential benefits of a strategy combining task interleaving and overlapping processing to optimize multitasking performance. Perhaps this potential can be even more exploited if tasks have to be performed which better support overlapping processing than the ones used in our experiments.

Altogether, then, the results raise at least as many questions as answers are provided. Three different sets of follow-up questions directly emerge from this research.

The first one has already mentioned above and concerns possible relationships between the preferred strategy of task organization when coping with multiple task demands and differences in other cognitive functions, i.e. WMC or intelligence. Furthermore, one might ask 
to what extend the finding of individual preferences for serial vs. parallel processing found in the current research is related to the concept of polychronicity. Originally, polychronicity was introduced as an anthropological concept to classify cultures with respect to what extent they value the performance of more than one task at a time (Tall, 1959). For the last 15 years, however, it has become a popular concept used to describe an individual preference for multitasking as opposed to perform one task at a time (monochronicity) in applied organizational and work contexts (e.g. Slocombe \& Bluedorn, 1999; Poposki \& Oswald, 2010). The preference for serial vs. overlapping processing in basic tasks like those used in the present experiments might reflect a basic cognitive correlate of this distinction.

The second set of questions concerns a better understanding of the task characteristics that make task interleaving strategies more efficient than serial processing and vice versa. Task interleaving strategies providing options for overlapping processing should be particularly efficient if this preferred style of multitasking is supported by the tasks to be performed concurrently. Two aspects might be relevant in this respect. The first one involves the similarity of two tasks with respect to their demands on processing resources. According to the multiple resource model of Wickens $(1984,2002)$ tasks can in principle be processed in parallel but this should be easier the less two tasks demand the same processing resources. Thus, the relative efficiency of an overlapping strategy compared to a strategy of serial processing should directly depend on the similarity of resource demands of the two tasks. A second possible factor relates to the specific structure of tasks. For example, task interleaving strategies might be particularly efficient and even more efficient than strict serial strategies if the tasks to be performed concurrently provide some natural breakpoints which support task interleaving (Janssen, Brumby \& Garrett, 2012). In addition, also aspects of the specific time structure of tasks might be relevant in this aspect (Katidioti \& Taatgen, 2014).

Finally, a third set of follow-up questions concerns the adaptivity and plasticity of the preferred strategies of multitasking. Evidence for a flexible use of serial vs. parallel processing has been provided in PRP research (e.g. Huebner \& Lehle, 2007). This directly raises the questions to what extent humans are able to adapt their strategy of multitasking most effectively to the task demands and how shifts between the different modes are performed (Fischer \& Plessow, 2015). Our findings suggest that such adaptivity also depends on the naturally preferred mode of multitasking. Individuals naturally preferring a task interleaving and/or overlapping mode are probably more flexible and effective in this respect than those preferring a serial mode. Closely linked to the question of adaptivity is the question of plasticity of preferred modes of multitasking. As to our knowledge, only one study, thus far, has ever addressed to what extend naturally preferred strategies of multitasking can be changed in the course of training (Damos et al., 1983). The results suggest that although participants might adapt a new strategy that deviates from the naturally preferred one, they might be able to do so only at the expense of performance decrements. This was particularly true for a subgroup of participants who were required to change from a strict serial "massed" strategy of processing of two discrete cognitive tasks to a simultaneous response strategy involving task interleaving. A better understanding of these issues involved in changing preferred styles of multitasking seems to be highly relevant for optimizing multitasking performance through specific trainings.

Providing the participants with more degrees of freedom for task organization and response scheduling was an important requirement to identify different personal preferences for coping with the multiple task requirements. However, an obvious drawback of this approach is that 
the underlying executive processes and the exact nature of serial versus parallel processes involved in the different strategies cannot precisely be disentangled. This holds specifically true for the paradigm of self-organized concurrent dual-task performance that was used in the second experiment. Any conclusions about the nature of performance strategies have to be based on post-hoc analyses of the response pattern produced by the individuals. This makes this paradigm probably prone to serious critic from a strict experimental perspective. However, the paradigms used in this research were chosen as good compromises providing sufficient degrees of freedom for task processing, but still having a necessary degree of control for detailed analyses of response timing and response pattern. New insights in human strategies on coping with multiple task requirements could be drawn by these paradigms and make it a promising approach for future research.

\section{Acknowledgments}

This research was partially supported by grant DFG 3759/4-1 provided from the Deutsche Forschungsgemeinschaft to the second author. Thanks are due to Jovita Bruening and Markus Janczyk for helpful comments to an earlier draft of this article, and to Marcus Bleil and Christian Dix for their technical support.

\section{References}

Altman, E.M. \& Trafton, J.G. (2002). Memory for goals: an activation-based model. Cognitive Science, 26, 39-83. doi: 10.1207/s15516709cog2601_2

Arrington, C. M., \& Logan, G. D. (2004). The cost of a voluntary task switch. Psychological Science, 15(9), 610-615. doi:10.1111/j.0956-7976.2004.00728.x

Arrington, C. M., \& Logan, G. D. (2005). Voluntary Task Switching: Chasing the Elusive Homunculus. Journal of Experimental Psychology: Learning, Memory, and Cognition, 31(4), 683-702. doi:10.1037/0278-7393.31.4.683

Bosman, E. A. (1993). Age-related differences in the motoric aspects of transcription typing skill. Psychology and Aging, 8, 87-102. doi:10.1037/0882-7974.8.1.87

Damos, D. L., \& Wickens, C. D. (1980). The identification and transfer of timesharing skills. Acta Psychologica, 46(1), 15-39. doi:10.1016/0001-6918(80)90057-8

Damos, D. L., Smist, T. E. \& Bittner, A. C. (1983). Individual differences in multiple-task performance as a function of response-strategy. Human Factors, 25, 215-226. doi:10.1177/001872088302500208

Fischer, R. \& Plessow, F. (2015). Efficient multitasking: parallel versus serial processing of multiple tasks. Frontiers in Psychology, 6: 1366. doi: 10.3389/fpsyg.2015.01366

Hall, E.T. (1959). The silent language. Garden City: Doubleday.

Huebner, R., \& Lehle, C. (2007). Strategies of flanker coprocessing in single and dual tasks. Journal of Experimental Psychology: Human Perception and Performance, 33, 103-123. doi:10.1037/0096-1523.33.1.103. 
Janczyk, M. (2015). Sequential modulation of backward crosstalk and task-shielding in dualtasking. Journal of Experimental Psychology: Human Perception and Performance, in press. doi: $10.1037 / x h p 0000170$

Janssen, C. P., Brumby, D. P. \& Garrett, R. (2012). Natural break points: The influence of priorities and cognitive and motor cues on dual-task interleaving. Journal of Cognitive Engineering and Decision Making, 6, 5-29. doi:10.1177/1555343411432339

Jastrow, J. (1891). The interference of mental processes - a preliminary survey. American Journal of Psychology, 4, 219-223.

Jersild, A. T. (1927). Mental set and shift. Archives of Psychology, 89.

Kane, M. J., Conway, A. R. A., Hambrick, D. Z. \& Engle, R. W. (2007). Variation in working memory capacity as variation in executive attention and control. In: Jarrold (ed.), Variation in working memory. New York: Oxford University Press.

Kane, M. J. \& Engle, R. W. (2000). Working memory capacity, proactive interference and divided attention: Limits on long-term memory retrieval. Journal of Experimental Psychology: Learning, Memory and Cognition, 26, 333-358. doi:10.1037/0278-7393.26.2.336

Kane, M. J. \& Engle, R. W. (2003). Working memory capacity and the control o attention: The contribution of goal neglect, response competition, and task set to Stroop interference. Journal of Experimental Psychology: General, 132, 47-70. doi: 10.1037/0096-3445.132.1.47

Katidioti, I. \& Taatgen, N.A. (2014). Choice in Multitasking: How Delays in the Primary Task Turn a Rational Into an Irrational Multitasker. Human Factors, 56(4), 728-736. doi: $10.1177 / 0018720813504216$

Kiesel, A., Steinhauser, M., Wendt, M., Falkenstein, M., Jost, K., Philipp, A. M., \& Koch, I. (2010). Control and interference in task switching—A review. Psychological Bulletin, 136(5), 849-874. doi:10.1037/a0019842

Lehle, C. \& Huebner, R. (2009). Strategic capacity sharing between two tasks: evidence from tasks with the same and with different task sets. Psychological Research, 5, 707-726. doi:10.1007/s00426-008-0162-6

Lehle, C., Steinhauser, M. \& Huebner, R. (2009). Serial and parallel processing in dual-tasks: What is more effortful? Psychophysiology, 46, 502-509. doi: 10.1111/j.14698986.2009.00806.x

Manzey, D. (1993). Doppelaufgabeninterferenz: Neue theoretische und methodische Perspektiven für ein altes Paradigma. In R. Daugs \& K. Blischke (Eds.), Aufmerksamkeit und Automatisierung in der Sportmotorik (pp. 79-96). Sankt Augustin: Academia.

Meyer, D. E., \& Kieras, D. E. (1997). A computational theory of executive cognitive processes and multiple-task performance: Part I. Basic mechanisms. Psychological Review, 104(1), 3-65. doi:10.1037/0033-295X.104.1.3

Meyer, D. E., Kieras, D. E., Lauber, E., Schumacher, E. H., Glass, J., Zurbriggen, E., Gemeindl, L., \& Apfelblat, D. (1995). Adaptive executive control: Flexible multiple-task 
performance without pervasive immutable response-selection bottlenecks. Acta Psychologica, 90(1-3), 163-190. doi:10.1016/0001-6918(95)00026-Q

Miller, J., Ulrich, R., \& Rolke, B. (2009). On the optimality of serial and parallel processing in the psychological refractory period paradigm: effects of the distribution of stimulus onset asynchronies. Cognitive psychology, 58(3), 273-310. doi:10.1016/j.cogpsych.2006.08.003

Monsell, S. (2003). Task switching. Trends in Cognitive Sciences, 7(3), 134-140. doi:10.1016/S1364-6613(03)00028-7

Navon, D., \& Gopher, D. (1979). On the economy of the human-processing system. Psychological Review, 86(3), 214-255. doi:10.1037/0033-295X.86.3.214

Navon, D. \& Miller, J. (1987). Role of outcome conflict in dual-task interference. Journal of Experimental Psychology: Human Perception and Performance, 13, 435-448. doi: 10.1037/0096-1523.13.3.435

Navon, D. \& Miller, J. (2002). Queing or sharing? A critical evaluation of the single-bottleneck notion. Cognitive Psychology, 44, 193-251. doi:10.1006/cogp.2001.0767

Pashler, H. (1994a). Dual-task interference in simple tasks: Data and theory. Psychological Bulletin, 116(2), 220-244. doi:10.1037/0033-2909.116.2.220

Pashler, H. (1994b). Graded capacity sharing in dual-task interference? Journal of Experimental Psychology: Human Perception and Performance, 20, 330-342. doi:10.1037/0096-1523.20.2.330

Pashler, H. (1994c). Overlapping mental operations in serial performance with preview. The Quarterly Journal of Experimental Psychology Section A, 47, 161-191. doi:10.1080/14640749408401148

Pashler, H., \& Johnston, J. C. (1989). Chronometric evidence for central postponement in temporally overlapping tasks. The Quarterly Journal of Experimental Psychology Section A, 41(1), 19-45. doi:10.1080/14640748908402351

Pashler, H. E. (2000). Task switching and multitask performance. In S. Monsell \& J. Driver (Eds.), Control of cognitive processes. Attention and Performance XVIII (pp. 277-307). Cambridge, Mass. [u.a.]: MIT Press.

Plessow, F. , Schade, S., Kirschbaum, C. \& Fischer, R. (2012). Better not to deal with two tasks at the same time when stressed? Acute psychosocial stress reduces task shielding in dual-task performance. Cognitive, Affective, \& Behavioral Neuroscience, 12, 557-570. doi: 103758/s13415-012-0098-6

Poposki, E.M. \& Oswald, F.L. (2010). The Multitasking Preference Inventory: Toward an improved measure o individual differences in polychronicity. Human Performance, 23, 247264.doi: 10.1080/08959285.2010.487843.

Rubin, O., \& Meiran, N. (2005). On the Origins of the Task Mixing Cost in the Cuing Task switching Paradigm. Journal of Experimental Psychology: Learning, Memory, and Cognition, 31(6), 1477-1491. doi:10.1037/0278-7393.31.6.1477 
Ruiz Fernández, S., Leonhard, T., Rolke, B., \& Ulrich, R. (2011). Processing two tasks with varying task order: Central stage duration influences central processing order. Acta Psychologica, 137(1), 10-17. doi:10.1016/j.actpsy.2011.01.016

Salthouse, T. A. (1985). Anticipatory processing in transcription typing. Journal of Applied Psychology, 70(2), 264-271. doi:10.1037/0021-9010.70.2.264

Schubert, T. (1996). Die Analyse von Interferenzeffekten bei der gleichzeitigen Bearbeitung zweier Aufgaben. [The analysis of interference effects in dual-tasks]. Zeitschrift für Psychologie, 4, 625-656.

Schumacher, E. H., Seymour, T. L., Glass, J. M., Fencsik, D. E., Lauber, E. J., Kieras, D. E., Meyer, D. E. (2001). Virtually perfect time sharing in dual-task performance: uncorking the central cognitive bottleneck. Psychological Science, 12(2), 101-108. doi:10.1111/14679280.00318

Slocombe, T.E. \& Bluedorn, A.C. (1999). Organizational behavior implications of the congruence between preferred polychronicity and experienced work-unit polychronicity. Journal of Organizational Psychology, 20, 75-99.

Spector, A., \& Biederman, I. (1976). Mental Set and Mental Shift Revisited. The American Journal of Psychology, 89(4), 669. doi:10.2307/1421465

Telford, C. W. (1931). The refractory phase of voluntary and associative responses. Journal of Experimental Psychology, 14(1), 1-36. doi:10.1037/h0073262

Tombu, M., \& Jolicœur, P. (2003). A central capacity sharing model of dual-task performance. Journal of Experimental Psychology: Human Perception and Performance, 29(1), 3-18. doi:10.1037/0096-1523.29.1.3

Trafton, G. J. \& Monk, C. A. (2008). Task interruptions. In: D.A. Boehm-Davis (ed.), Reviews of human factors and ergonomics, Vol. 3 (pp. 111-126). Santa Monica: Human Factors and Ergonomics Society.

Ulrich, R., \& Miller, J. (2008). Response grouping in the psychological refractory period (PRP) paradigm: Models and contamination effects. Cognitive Psychology, 57(2), 75-121. doi:10.1016/j.cogpsych.2007.06.004

Wickens, C. D. (1984). Processing resources in attention. In R. Parasuraman \& R. Davies (Eds.), Varieties of attention (pp. 63-101). New York: Academic Press.

Wickens, C. D. (2002). Multiple resources and performance prediction. Theoretical Issues in Ergonomics Science, 3(2), 159-177. doi:10.1080/14639220210123806

Wickens, C. D., \& McCarley, J. S. (2008). Applied Attention Theory. Boca Raton: CRC Press.

Wickens, C. D., Mountford, S. J. \& Schreiner, W. (1981). Multiple resources, taskhemispheric integrity, and individual differences in time-sharing. Human Factors, 23, $211-$ 229. doi: $10.1177 / 001872088102300209$

Wickens, C. D., Gutzwiller, R. S. \& Santamaria, A. (2015). Discrete task switching in overload: A meta-analyses and model. International Journal of Human-Computer Studies, 79, 79-84. doi:10.1016/j.ijhcs.2015.01.002 